ESAIM: PROCEEDINGS, February 2007, Vol.16, 89-113

Eric Cancès \& Jean-Frédéric Gerbeau, Editors

DOI: $10.1051 /$ proc:2007010

\title{
TOWARDS HYBRID LES-RANS-COUPLING FOR COMPLEX FLOWS WITH SEPARATION*
}

\author{
Jaffrézic B. ${ }^{1}$, Breuer M. ${ }^{1}$, Chikhaoui O. ${ }^{2}$, Deng G. ${ }^{2}$ And Visonneau M. ${ }^{2}$
}

\begin{abstract}
The paper is concerned with a new hybrid LES-RANS approach, which splits up the simulation into a near-wall RANS part and an outer LES part, both modes relying on a one-equation model for the turbulent kinetic energy. This model has been tested on the standard plane channel flow test case as well as on the flow over a periodic arrangement of hills. Encouraging results were achieved. In an additional study based on the detached-eddy simulation (DES) concept attempts have been made to improve the accuracy of the simulation by using adaptive local grid refinement. For this purpose, a criterion based on the residual of the budget of the mean momentum equations has been studied.
\end{abstract}

\section{INTRODUCTION}

Large-eddy simulation (LES) is a highly promising technique for the prediction of complex turbulent flows including large-scale flow phenomena such as massive separation, large recirculation regions and vortex shedding [6]. However, LES still suffers from the matter of fact that most turbulent flows of practical relevance cannot be computed because of the extremely large resources required, especially for high Reynolds number flows encountered in technical applications. In order to go beyond the present state-of-the-art, extremely fine grids required for the resolution of the near-wall region (e.g., thin boundary layers) have to be avoided.

For this purpose a hybrid LES-RANS approach is developed, which tries to split up the simulation (ideally not the domain) into a RANS part and an LES part. Typically, RANS is adequate for attached boundary layers requiring reasonable CPU-time and memory, where LES can also be applied but demands extremely large resources. Contrarily, RANS often fails in flows with massive separation and/or with large-scale vortical structures. Here, LES is without doubt the best choice. The basic concept is to combine the advantages of both methods yielding an optimal solution at least for a special class of flows. A real non-zonal hybrid technique is preferred since it avoids the predefinition of RANS and LES regions leading to an approach where the suitable simulation technique is chosen more or less automatically. This is a big advantage compared to Detached-Eddy Simulation (DES) formulated by Spalart [27]. Although DES is claimed to be a non-zonal approach by the authors, a close look at the switching criterion shows that the interface location is closely related to the grid design and thus predefined by the user. The hybrid strategy proposed in this paper avoids this grid dependency

* The project is financially supported by the Deutsche Forschungsgemeinschaft (BR 1847/8-1) and the Centre National de la Recherche Scientifique within the French-German Programme 'LES for complex flows' (FOR 507). The computations were partially carried out at RRZE Erlangen, LRZ München, IDRIS and CINES. The organization of CEMRACS 2005 and all kinds of support are gratefully acknowledged.

${ }^{1}$ Institute of Fluid Mechanics, University of Erlangen-Nürnberg, Cauerstr. 4, D-91058 Erlangen, Germany;

e-mail: bjaffrez@lstm.uni-erlangen.de \& breuer@lstm.uni-erlangen.de

2 Ecole Centrale de Nantes, Laboratoire de Mécanique des Fluides CNRS UMR 6598, Nantes, France;

e-mail: Oussama.Chikhaoui@ec-nantes.fr \& Ganbo.Deng@ec-nantes.fr \& Michel.Visonneau@ec-nantes.fr

(c) EDP Sciences, SMAI 2007 
by defining the interface based on physical issues (see Section 1.3). Although the idea of combined LES-RANS methods is not new, a variety of open questions has to be answered. This includes in particular the demand for appropriate coupling techniques between LES and RANS, adaptive control mechanisms, and proper SGS/RANS models.

Additionally, a study has been carried out on a local grid refinement approach. Indeed, in LES systematic refinement is usually adopted to solve the problem of insufficient spatial resolution leading to extremely fine grids, especially in the very expensive near-wall region. Thus a choice for a local grid refinement criterion based on mean quantities provided directly by the simulations is proposed. This approach is assumed to be an interesting issue for improvements of the spatial resolution without excessive resource requirements.

In order to investigate the above mentioned open questions, the 'flow over periodic hills' test case including pressure-induced flow separation and subsequent reattachment is considered in addition to the standard plane channel flow test case.

\section{HYBRID LES-RANS METHOD}

A variety of different hybrid concepts were proposed in the literature. A crude distinction between various proposals is given by zonal and non-zonal techniques. In the first case, the user predefines the LES and RANS regions, which is often difficult to carry out for unknown flows. Conversely, the non-zonal approach chooses (more or less) automatically the suitable simulation technique and thus avoids the region predefinition. Here a gradual transition between both methods takes place which weakens the problem of setting up an appropriate coupling strategy between RANS and LES zones not available today. Hence in the present study a non-zonal approach is preferred. Another important issue is the question of suitable models for such hybrid methods. For the non-zonal methodology a unique model is more advantageous. In the context of eddy-viscosity models for RANS, a two-equation model is a natural choice since one transport equation is solved for the velocity scale and one for the length scale. For LES zero- or one-equation models are more obvious since the length scale is naturally given by the filter width $\Delta$. Consequently, these facts are in contradiction to a unique model. However, if the near-wall region is the main target for RANS, the length scale can be prescribed by an algebraic relation. This leads to a one-equation model for both zones.

For the near-wall RANS region the model adopted in the present study is based on the two-layer approach proposed by Rodi et al. [23]. They used a one-equation model for the viscosity-affected near-wall layer and combined it with a standard $k-\epsilon$ model for the outer region to a two-layer RANS model. Hence, the formulation for the inner layer is taken over, but the outer region is basically replaced by LES. The resulting unique model consists of a transport equation for the modeled turbulent kinetic energy $k_{\text {mod }}$ in the RANS mode and the subgrid scale (SGS) turbulent kinetic energy $k_{s g s}$ in the LES mode, respectively. The two models are then distinguished by their definitions of the dissipation rate $\epsilon$ of the turbulent kinetic energy and the eddy-viscosity $\nu_{t}$ (see Sections 1.1 and 1.2).

The general transport equation for $k$ reads:

$$
\begin{aligned}
\frac{\partial k}{\partial t}+\overline{U_{j}} \frac{\partial k}{\partial x_{j}} & =\frac{\partial}{\partial x_{j}}\left[\left(\nu+\frac{\nu_{t}}{\sigma_{k}}\right) \frac{\partial k}{\partial x_{j}}\right]+\nu_{t}\left(\frac{\partial \overline{U_{i}}}{\partial x_{j}}+\frac{\partial \overline{U_{j}}}{\partial x_{i}}\right) \frac{\partial \overline{U_{i}}}{\partial x_{j}}-\epsilon \\
\text { with } \sigma_{k} & =1.0
\end{aligned}
$$

$\overline{U_{i}}$ has to be understood as time-averaged $U_{i}$ in RANS mode and filtered $U_{i}$ in LES mode.

\subsection{SGS model for the hybrid technique}

Before applying the new hybrid LES-RANS technique, it was necessary to agree on a subgrid scale model for the LES mode. The basic idea is to employ a similar one-equation model for $k$ in RANS and LES modes. 
The standard formulation of $\epsilon$ and $\nu_{t}$ for the SGS one-equation model $[21,24,25,29]$ reads:

$$
\begin{aligned}
\nu_{t} & =C_{\mu} k_{\text {sgs }}^{1 / 2} \Delta \\
\epsilon & =C_{d} k_{\text {sgs }}^{3 / 2} / \Delta \\
\Delta & =(\Delta x \Delta y \Delta z)^{1 / 3}
\end{aligned}
$$

Thus the decision had to be taken on the value of the constants $C_{d}$ and $C_{\mu}$ used for the definition of $\epsilon$ and $\nu_{t}$, respectively. For this purpose several sets of constants have been tested on the plane channel flow test case. The tests were performed applying constants suggested by Schumann [25], Yoshizawa [29] and Pope [21] relying on the Kolmogorov energy spectrum as well as a model proposed by Sagaut [24] also based on the energy spectrum but using spectral methods such as EDQNM (Eddy-Damped Quasi-Normal Markovian) theory for the adjustment of the constants. All models gave similar results. Therefore, it has been decided that the main set should be $C_{\mu}=0.05$ and $C_{d}=1.0$ as proposed by Schumann. However, as will be seen in Section 5.2 the LES models have to be checked for more complex flow situations and under varying grid resolutions.

\subsection{RANS model for the hybrid technique}

The next step of the study was to define the RANS model which is applied in the hybrid LES-RANS technique. The objective was to use a simple model based on the turbulent kinetic energy and due to the restricted area in which the model operates, especially designed for the near-wall region. Moreover, it is preferred to avoid the disadvantage of using damping functions, which are normally required for the length scale $l_{\mu}$ and the dissipation length $l_{\epsilon}$. One model meets these mentioned key points.

In order to improve the quality of the prediction of the RANS technique, Rodi et al. [23] formulated a oneequation near-wall turbulence model. The particularity of the model is the use of the wall-normal velocity fluctuations $\overline{v^{\prime 2}}$ as the velocity scale instead of $k$. As a consequence only one damping function is required. The formulation shown below is the original model suggested by Rodi et al. [23], which was combined with the $k-\epsilon$ model in the outer region to a two-layer RANS approach.

The empirical relations for $\nu_{t}$ and $\overline{v^{\prime 2}}$ read:

$$
\begin{aligned}
\nu_{t} & =\left(\overline{v^{\prime 2}}\right)^{1 / 2} l_{\mu, v} \\
l_{\mu, v} & =C_{l, \mu} y \\
\overline{v^{\prime 2}} / k_{\text {mod }} & =4.65 \times 10^{-5} y^{* 2}+4.00 \times 10^{-4} y^{*} \quad \text { for } \quad y^{*} \leq 60 \\
y^{*} & =k_{\text {mod }}^{1 / 2} y / \nu \\
C_{l, \mu} & =0.33
\end{aligned}
$$

The empirical relations for $\epsilon$ are given by:

$$
\begin{aligned}
\epsilon & =\left(\overline{v^{\prime 2}}\right)^{1 / 2} k_{\text {mod }} / l_{\epsilon, v} \\
l_{\epsilon, v} & =1.3 y /\left(1+2.12 \frac{\nu}{\left(\overline{v^{\prime 2}}\right)^{1 / 2} y}\right)
\end{aligned}
$$

As shown by Rodi et al. [23] and originally introduced by Durbin [14], for the near-wall region (RANS mode) the wall-normal velocity fluctuations $\left(\overline{v^{\prime 2}}\right)^{1 / 2}$ are better suited to characterize the turbulent motion than using $k$ as the characteristic velocity scale. The length scale $l_{\mu, v}$ appearing in the eddy-viscosity relation $\nu_{t}$, generally defined as $\nu_{t}=C_{\mu} k^{1 / 2} l_{\mu}$ in a one-equation model now scales linearly with the wall distance $y$ and needs no damping function anymore. Indeed the eddy-viscosity reduction in the vicinity of the wall is an effect of the decreasing $v^{\prime}$ fluctuations rather than a viscous one and hence is naturally taken into account. The dissipation 
length $l_{\epsilon, v}$ used to define the dissipation rate $\epsilon$, which usually reads $\epsilon=C_{d} k^{3 / 2} / l_{\epsilon}$ in a one-equation model, also scales linearly near the wall. Only in the immediate vicinity of the wall the distribution must be modified to yield the correct behavior of $\epsilon \sim y^{0}$ as $y$ goes to zero. In order to apply $\left({\overline{v^{\prime}}}^{2}\right)^{1 / 2}$ as the velocity scale in the model, Rodi et al. provided an equation to relate the wall-normal velocity fluctuations to the distribution of $k$ (equivalent to $k_{m o d}$ in RANS mode) so that the transport equation does not have to be adjusted. Instead of using $y^{+}$, formulated with $u_{\tau}$, they introduced $y^{*}$, which allows the model to be applied in separated flows, for which $\tau_{w}$ is zero or negative. This formulation of $\overline{v^{\prime 2}}$ is valid up to $y^{*} \approx 60$, which according to DNS results [18] corresponds to $y^{+} \approx 30$. Finally, one can notice that the constant $C_{\mu}$ usually used in the eddy-viscosity definition is now included in the eddy-viscosity length scale relation.

\subsection{LES-RANS interface}

Two critical points are how to combine both techniques and how to choose a criterion to shift from RANS to LES. In Rodi et al.'s two-layer approach [23], the ratio of the eddy-viscosity to the molecular viscosity $\nu_{t} / \nu$ is employed as switching criterion. In the vicinity of the wall, the one-equation near-wall turbulence model is applied until the ratio reaches a fixed value $C_{\text {switch }}$. Then the outer model is used. In order to keep the requirement $y^{*} \leq 60, C_{\text {switch }}$ was set to 16 by Rodi et al. [23]. In the hybrid LES-RANS technique this value cannot be applied as $\nu_{t}$ in the LES mode is much lower than $\nu_{t}$ in the RANS mode. Furthermore, once the critical value of $\nu_{t}\left(\left(\nu_{t}\right)_{\text {crit }}=\nu \cdot C_{\text {switch }}\right)$ is attained in the RANS mode, $\nu_{t}$ will strongly decrease in the LES mode and hence fall below the critical value $\left(\nu_{t}\right)_{\text {crit }}$ again. Then the entire simulation is treated in RANS what is not desired. The suitable ratio for the LES-RANS switch is found for $C_{\text {switch }}<1$. However, this criterion did not offer suitable results. Although the simulation shifts from RANS to LES at a reasonable distance from the wall, the technique switches back from LES to RANS in the core of the domain when $\nu_{t}$ decreases and finally reaches the critical ratio $\left(\nu_{t}\right)_{c r i t}$. Therefore, this criterion has been abandoned. As a remedy for the present hybrid LES-RANS technique, different dynamic switching criteria have been tested to define the interface between RANS and LES:

- Validity restriction of $\left(\overline{v^{\prime 2}}\right)$ :

$$
y^{*} \leq 60=C_{\text {switch }}
$$

Here the dynamic criterion $y^{*}$ based on $k$ is applied to define the LES-RANS interface. This condition gives, in the channel flow test case, no real delimited LES-RANS regions (see Fig. 1 and the following criterion $y_{2}^{*}$ for explanation). The hybrid LES-RANS technique using the present criterion is named 'hybrid version $A$ '.

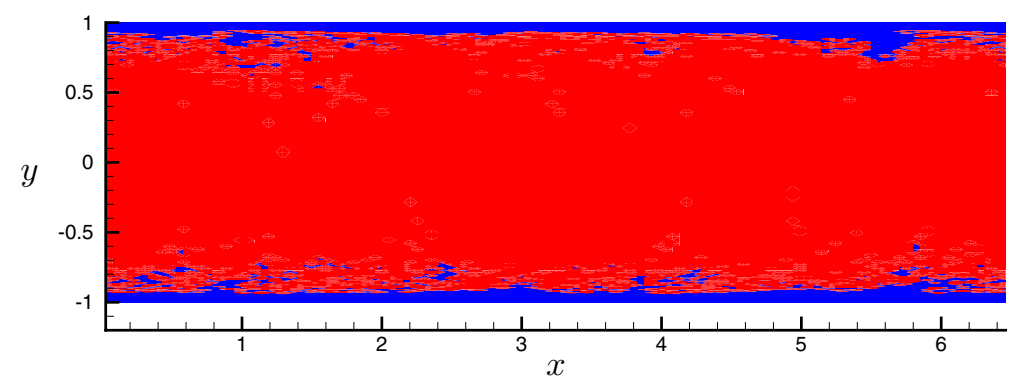

Figure 1: Channel flow test case at $R e_{\tau}=590$. RANS-LES domain layout. RANS domain: blue zone. LES domain: red zone. The blue islands in the core region are undesired. 

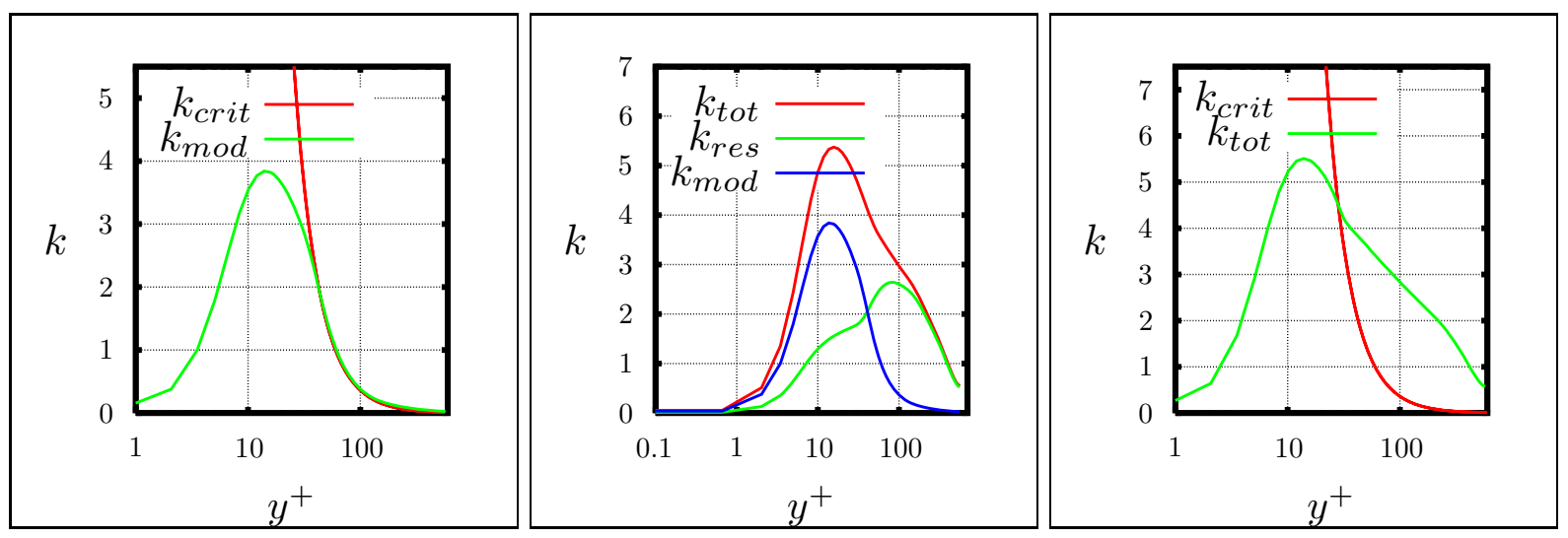

Figure 2: Results for the channel flow test case at $R e_{\tau}=590$. Behavior of $k_{m o d}$ in comparison with $k_{\text {crit }}$ (left). $k_{m o d}$, $k_{r e s}$ and $k_{t o t}$ of hybrid version $A$ (center). Behavior of $k_{t o t}$ used in $y_{2}^{*}$ in comparison with $k_{\text {crit }}$ in hybrid version $B$ (right).

- New criterion $y_{2}^{*}$ :

$$
y_{2}^{*} \leq 60=C_{\text {switch }}
$$

In order to get over the last remark, the new criterion $y_{2}^{*}$ is introduced, which is defined as follows $y_{2}^{*}=k_{t o t}^{1 / 2} y / \nu$. Hence $y_{2}^{*}$ is a modification of $y^{*}$ by replacing $k_{m o d}$ by $k_{t o t}=k_{m o d}+k_{r e s}$. Here $k_{t o t}$ and $k_{r e s}$ denote the total and the resolved turbulent kinetic energy, respectively. After testing $y^{*}$, it was seen that no clearly delimited RANS and LES regions were obtained. This fact is a consequence of $k_{m o d}$ used in the formulation of $y^{*}$. As can be seen in Fig. 2 (left), $k_{m o d}$ used in 'hybrid version $A^{\prime}$ closely follows the curve $k_{\text {crit }}$, which is defined as $k_{\text {crit }}=\left(C_{\text {switch }} \nu / y\right)^{2}$ and represents the limit between RANS and LES ( $k_{\text {mod }} \leq k_{\text {crit }}$ meaning RANS mode). As a remedy the presence of resolved turbulent scales in the RANS region (see Fig. 2, center) is accounted for. Indeed, in steady RANS $k_{m o d}$ is expected to correspond to $k_{t o t}$ and thus $k_{r e s}$ should be zero. That is not the case here. Since it operates as an unsteady RANS (URANS), also the RANS mode resolves some turbulent scales. Thus $k_{\text {mod }}$ in $y^{*}$ is replaced by $k_{t o t}$, which gives a higher value of $k$ used in the switching criterion and by this way is assumed to provide two distinct RANS and LES domains. The effect of this modification is observed in Fig. 2 (right). Unlike $y^{*}, y_{2}^{*}$ provides a sharp interface between RANS and LES.

It has to be noticed that the condition $y_{2}^{*} \leq 60$ always keeps the requirement $y^{*} \leq 60$ and that $y^{*}$ is kept in the formulation of the model. The LES-RANS interface using $y_{2}^{*} \leq 60$ as criterion is located at $y^{+} \approx 30$. The hybrid LES-RANS technique using the present criterion is named 'hybrid version $B$ '.

\subsection{Modified formulations of the RANS model}

\subsubsection{Adjustment of the RANS model to a higher Reynolds number.}

In order to match the objectives of the present study, i.e. to apply LES to high Reynolds number turbulent flows, the model performance has been compared with a more recent, higher Reynolds number $\left(R e_{\tau}=590\right)$ DNS of a channel flow (see Section 4.1) compared to the reference DNS used by Rodi $\left(R e_{\tau}=180\right.$ and 395$)$. This study shows that an adjustment of the constants is worth being tested (see Fig. 3). An enhanced RANS model using the same architecture but modified constants leading to a much better agreement between the modeled and the real distributions of $\overline{v^{\prime 2}} / k, l_{\mu, v}^{+}$and $C_{\mu}^{1 / 2} l_{\epsilon, v}^{+}$was defined. The range of interest for the RANS model is approximately $y^{+} \leq 30$. 

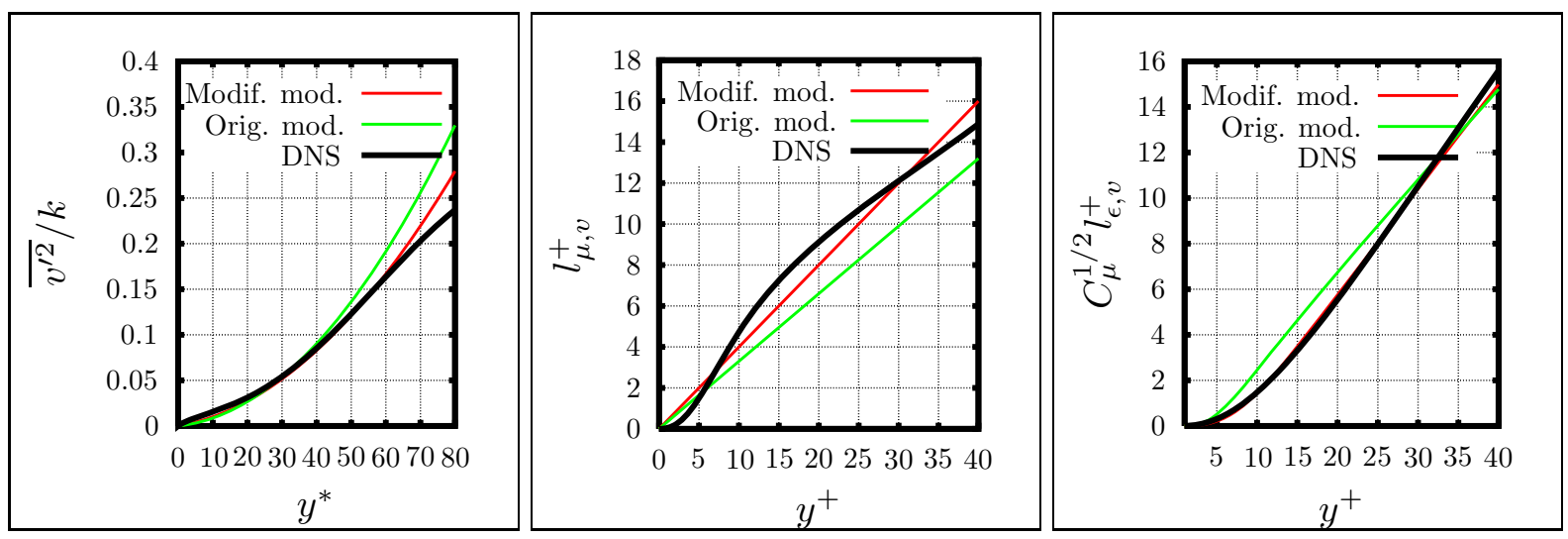

Figure 3: Channel Flow: Distribution of $\overline{v^{\prime 2}} / k$ (left), $l_{\mu, v}^{+}$(center) and $C_{\mu}^{1 / 2} l_{\epsilon, v}^{+}$(right). Modif. model: New model formulation using DNS results of Moser et al. [18] at $R e_{\tau}=590$. Orig. model: Rodi et al.'s formulation [23] based on low-Re results but plotted for DNS results of Moser et al. [18] at $R e_{\tau}=590$.

The new empirical relations for $\nu_{t}$ and $\epsilon$ read:

$$
\begin{aligned}
\overline{v^{\prime 2}} / k_{\text {mod }} & =3.55 \times 10^{-5} y_{2}^{* 2}+6.50 \times 10^{-4} y_{2}^{*} \quad \text { for } \quad y_{2}^{*} \leq 60 \\
C_{l, \mu} & =0.40 \\
l_{\epsilon, v} & =1.5 y /\left(1+7.65 \frac{\nu}{\left(\overline{v^{\prime 2}}\right)^{1 / 2} y}\right)
\end{aligned}
$$

This new set of constants can be applied to each previously described model versions $(A$ and $B)$, which are named hybrid versions $\mathrm{Am}$ and $\mathrm{Bm}$ when the new set of constants is employed.

\subsubsection{Adjustment of the RANS eddy-viscosity.}

This second modification is a consequence of the presence of resolved turbulent scales in the RANS region. Indeed, as previously mentioned (see Section 1.3) in steady RANS $\left(\overline{u_{i}^{\prime} u_{j}^{\prime}}\right)_{\text {mod }}$ corresponds to $\left(\overline{u_{i}^{\prime} u_{j}^{\prime}}\right)_{t o t}$ and the modeled eddy-viscosity $\nu_{t, R A N S}^{\text {mod }}$ provided by the model is the total eddy-viscosity $\nu_{t, R A N S}^{\text {tot }}$. Nevertheless, the first campaign of tests carried out on the hybrid approach (see Section 5.1.1) showed a different outcome with the pronounced presence of resolved turbulent scales in the RANS region. Under this condition $\nu_{t, R A N S}^{t o t}$ cannot be longer seen as $\nu_{t, R A N S}^{\text {mod }}$ but represents the sum of $\nu_{t, R A N S}^{\text {mod }}$ and a resolved eddy-viscosity $\nu_{t, R A N S}^{r e s}$. This $\nu_{t, R A N S}^{r e s}$ is obviously not explicitly calculated but depicts the effect of the resolved field.

According to this remark the important contribution of the resolved field might also be taken into account and the formulation of the RANS model, as part of the hybrid LES-RANS approach, be somehow adjusted.

In the RANS model, $\nu_{t, R A N S}^{\text {mod }}$ is calculated with $k_{\text {mod }}$, which is not $k_{t o t}$ because of the unsteady character of the simulation and thus lower than a corresponding $k_{m o d}$ computed in steady RANS. Therefore, the resolved field is already accounted for in the calculation of $\nu_{t, R A N S}^{\text {mod }}$. Nevertheless, the under-prediction of $U$ in the channel flow test case (see Fig. 4), which could be explained by the over-prediction of $\nu_{t, R A N S}^{t o t}$ seems to show that this automatic adjustment of the model is insufficient. This statement is confirmed by Fig. 5 (left), which presents $\nu_{t, R A N S}^{\text {mod }}$. In the RANS region the modeled $\nu_{t}$ closely follows $\nu_{t}$ provided by DNS results but representing the reference total eddy-viscosity $\nu_{t, R A N S}^{t o t}$. Therefore, it can be assumed that $\nu_{t, R A N S}^{t o t}$ in the case of the hybrid LES-RANS technique is over-predicted.

Thus, if the assumption that $\nu_{t, R A N S}^{\text {mod }}$ should still be adjusted according to the resolved field, one could notice that the contribution $\left(\overline{u^{\prime} v^{\prime}}\right)_{\text {res }}$ (Fig. 5, center) is not taken into account in the automatic eddy-viscosity adjustment 

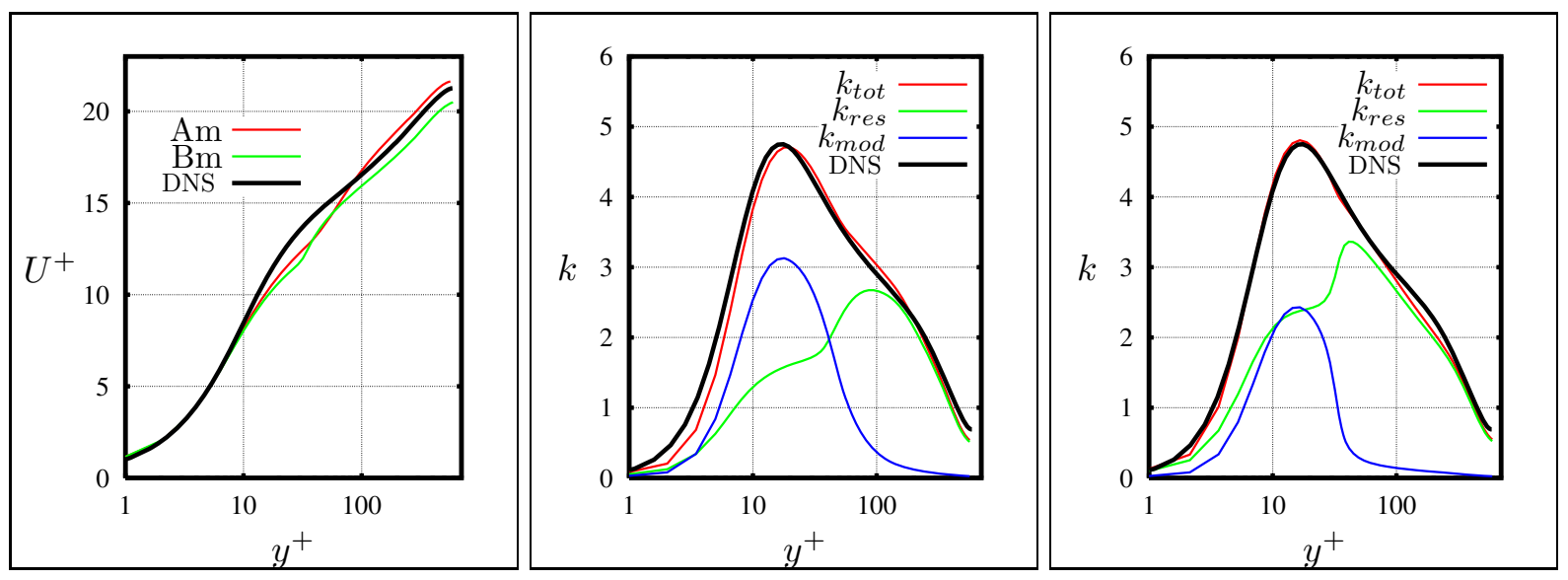

Figure 4: Results for the channel flow test case at $R e_{\tau}=590$. Predictions of the mean streamwise velocity $U^{+}$for the cases hybrid versions Am and Bm (left), $k$ for hybrid versions Am (center) and Bm (right). DNS result of Moser et al. [18].

by $k$. Additionally, the ratio $\left(\overline{u^{\prime} v^{\prime}}\right)_{r e s} /\left(\overline{u^{\prime} v^{\prime}}\right)_{t o t}$ versus $y_{2}^{*}$ (Fig. 5, right) obtained from the statistical results of the plane channel flow with hybrid version $B m$ never passes below 0.3 (value at the position $y_{2}^{*}=12$ or $y^{+} \approx 7$ ) in the relevant RANS region and reaches 0.65 close to the interface $\left(y_{2}^{*}=60\right.$ or $\left.y^{+} \approx 30\right)$. This result exhibits the importance of this resolved field in the RANS region and the persistency of the resolved scales which do not vanish as expected. The significant level of the resolved field is plausibly due to the position of the interface relatively close to the wall as well as the high resolution of the grid used (see Section 5.1). The questions arising now are: should $\nu_{t, R A N S}^{\text {mod }}$ be readjusted? If yes, should this adjustment be performed according to $\left(\overline{u^{\prime} v^{\prime}}\right)_{\text {res }}$ ? If yes, how should the resolved Reynolds shear stress field be 'introduced' in the RANS formulation?

Assuming that the two first questions can be answered positively, the remaining one is how to adjust the eddyviscosity. A natural choice to observe the importance of the resolved shear stress in the simulation is the ratio $\left(\overline{u^{\prime} v^{\prime}}\right)_{\text {res }} /\left(\overline{u^{\prime} v^{\prime}}\right)_{\text {tot }}$. A function providing this ratio could be an interesting starting point and the adjustment of $\nu_{t, R A N S}^{\text {mod }}$ could resemble to $\nu_{t, R A N S}^{\text {mod }}=\nu_{t, R A N S}^{\text {mod }} \cdot(1-f)$ with $f$ representing the effect of $\left(\overline{u^{\prime} v^{\prime}}\right)_{\text {res }}$.

In order to verify these assumptions, an empirical function $f\left(y_{2}^{*}\right)$ (with no character of generality) mimicing the ratio $\left(\overline{u^{\prime} v^{\prime}}\right)_{\text {res }} /\left(\overline{u^{\prime} v^{\prime}}\right)_{\text {tot }}$ (see Fig. 5, right) is formulated:

$$
\begin{gathered}
\begin{cases}f\left(y_{2}^{*}\right)=0.3 \cdot y_{2}^{*} & \text { for } \quad 0 \leq y_{2}^{*} \leq 1 \\
f\left(y_{2}^{*}\right)=0.3 & \text { for } \quad 1<y_{2}^{*} \leq 12 \\
f\left(y_{2}^{*}\right)=0.3+\left(1.55 \cdot 10^{-4} \cdot\left(y_{2}^{*}-12\right)^{2}\right) & \text { for } \quad 12<y_{2}^{*} \leq 60\end{cases} \\
\nu_{t, \text { RANS }}^{\text {mod }}=\nu_{t, \text { RANS }}^{\text {mod,temp }} \cdot\left(1-f\left(y_{2}^{*}\right)\right)
\end{gathered}
$$

This function $f\left(y_{2}^{*}\right)$ compensating for the presence of $\left(\overline{u^{\prime} v^{\prime}}\right)_{r e s}$ in the formulation of $\nu_{t, R A N S}^{\text {mod }}$ is used as follows. $\nu_{t, R A N S}^{\text {mod }}$ is first calculated as previously mentioned in hybrid version Bm providing $\nu_{t, R A N S}^{\text {mod,temp }}$. In a second step the function $f\left(y_{2}^{*}\right)$ is applied, giving the effective $\nu_{t, R A N S}^{\text {mod }}$. An important remark is that the function $f\left(y_{2}^{*}\right)$ is presently not dynamically predicted within the simulation. The present function is designed for hybrid version Bm; the new version is named hybrid version Bmf. In order to avoid the empirical character of the compensating function $f\left(y_{2}^{*}\right)$ and to allow for the prediction of more complex (separated) flows, a dynamic procedure for the determination of $f\left(y_{2}^{*}\right)$ was considered and presented in [2]. This dynamic compensating 

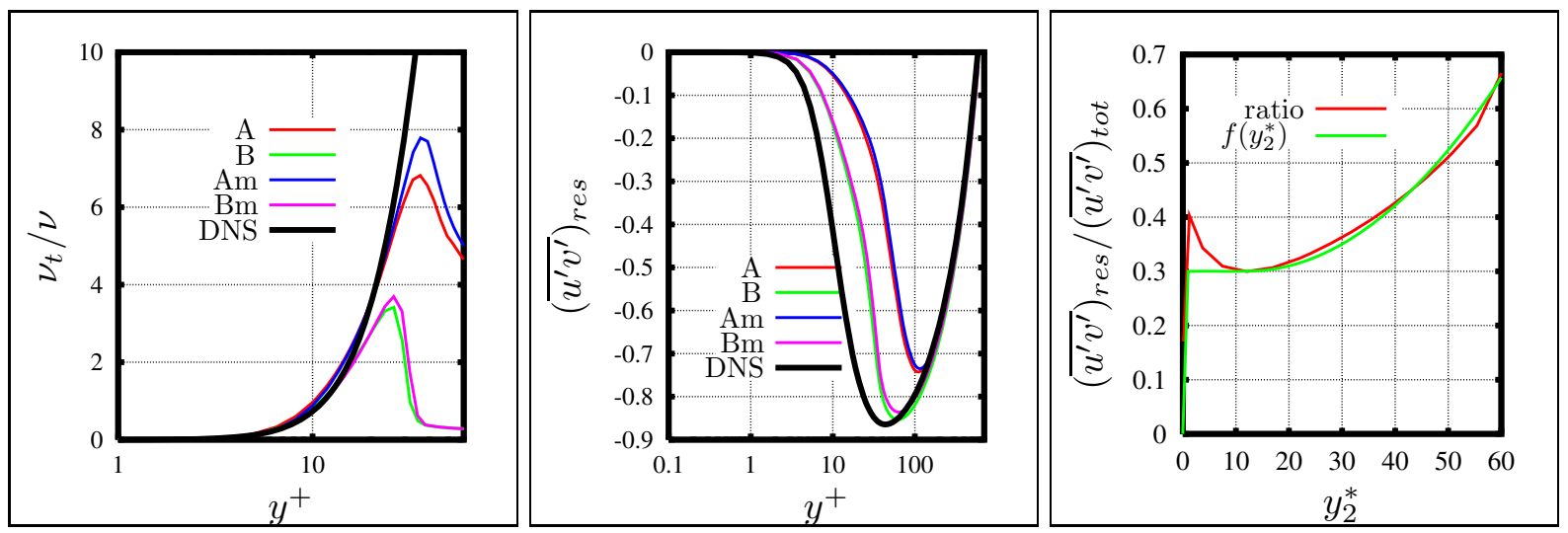

Figure 5: Results for the channel flow test case at $R e_{\tau}=590 . \quad \nu_{t, R A N S}^{\bmod } / \nu$ (left) and resolved Reynolds stresses $\overline{u^{\prime} v^{\prime}}$ (center) for the hybrid versions $A, B, A m$ and Bm. Ratio $\left(\overline{u^{\prime} v^{\prime}}\right)_{r e s} /\left(\overline{u^{\prime} v^{\prime}}\right)_{\text {tot }}$ for the hybrid version Bm and function $f\left(y_{2}^{*}\right)$ (right). LES-RANS interface at $y_{2}^{*}=60\left(y^{+} \approx 30\right)$ for versions $B$ and Bm.

function has provided equivalent improvements than the present empirical function $f\left(y_{2}^{*}\right)$ for which the results are shown in Section 5.1.3.

\section{AdAptive GRID REFinEMENT}

For LES and DES simulations it was found that the results are more sensitive to the grid quality and density compared with a RANS computation. Only a few articles in the literature deals with adaptive local grid refinement applied to these specific simulations. Generally, the solution used is a systematic refinement which increases considerably the required resources.

In order to improve the accuracy of LES and DES simulations, attempts to perform an adaptive grid refinement have been carried out in order to take benefit of the unstructured solver (see Section 3.2). The focus here has been to study the behavior of the residual of the budget for the mean momentum equations in DES to provide a criterion for local grid refinement. The grid and the results obtained using this criterion are presented for the case of the flow over the periodic hill in Section 5.4.

\section{Numerical Methodology}

The present study involves two different groups and the computations are performed applying two different codes. The first, namely $\mathcal{L E S O C C}$ is used by LSTM Erlangen, whereas the simulations by Ecole Centrale de Nantes are performed with the ISIS code.

\section{1. $\mathcal{L} \mathcal{E S O C C}$}

The LES code $\mathcal{L E S O C C}$ used for the solution of the filtered Navier-Stokes equations, is a $3-\mathrm{D}$ finite-volume solver for arbitrary non-orthogonal and non-staggered (block-structured) grids [4-6]. The spatial discretization of all fluxes is based on a central scheme of second-order accuracy. A low-storage multi-stage Runge-Kutta method (second-order accurate) is applied for time-marching. Beside the hybrid approach described above, different SGS models for LES are implemented (Smagorinsky model and dynamic Smagorinsky model) as well as DES (Spalart-Allmaras model [26]). These features are used to provide data for comparison with the new hybrid method. 


\subsection{ISIS}

To handle complex geometries efficiently, an unstructured code ISIS [10-12] is developed at Ecole Centrale de Nantes. It is a finite-volume code solving the Navier-Stokes equations for an incompressible fluid using arbitrary control volumes. The convective fluxes are discretized with a second-order central scheme or an upwind-blended scheme. A second-order central scheme is applied to the diffusion fluxes. A second-order backward differences implicit scheme is employed for time discretization. Pseudo-time stepping is used for steady flow computations. The Rhie and Chow approach [22] is employed to evaluate the mass flux in a nonstaggered layout. The velocity-pressure coupling is solved by a SIMPLE like algorithm. The pressure equation is solved with a preconditioned conjugate gradient solver. The code was initially designed for RANS simulation. Several statistical models ranging from one-equation Spalart-Allmaras, two-equation linear and non-linear models such as $k-\omega$ SST model and explicit algebraic stress model, to seven-equation differential Reynolds stress model are implemented and extensively validated [10-12]. ISIS was also validated successfully in the context of DNS, LES and hybrid LES/RANS (mainly DES) upon different classical test cases [9].

\section{Test Cases}

\subsection{Plane channel flow}

The first test case used for validating the basic settings and model modifications, is a plane channel flow with periodic boundary conditions in streamwise and spanwise directions and no-slip boundary condition at the walls. The dimensions are: $2 \pi$ (streamwise) $\times \pi$ (spanwise) $\times 2$ (wall-normal direction). The results of our tests are compared with DNS data of fully developed plane turbulent channel flow at a Reynolds number of $R e_{\tau}=590\left(R e_{b}=10,935\right)$ provided by Moser et al. [18].

\subsection{Periodic hill flow}

The hill flow configuration was a test case at the 'ERCOFTAC/IAHR/COST Workshop on Refined Turbulence Modeling' in 2001 and 2002 [16,17]. The basic idea is to set up a geometrical 'simple' test case which allows to perform basic investigations based on a 'complex' flow including pressure-induced separation and subsequent reattachment. Fig. 6 shows a sketch of the configuration. The dimensions of the domain are: $L_{x}$ (streamwise direction) $9.0 h, L_{y}$ (wall-normal direction) $3.035 h$ and $L_{z}$ (spanwise direction) $4.5 h$. The flow is assumed to be periodic in the streamwise direction. As no DNS was performed at the Reynolds number of $R_{e}=10,595$,

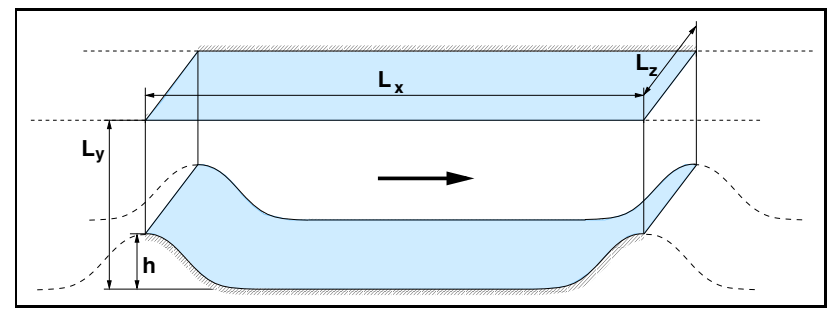

Figure 6: 3D sketch of the periodic hill flow test case.

the reference solution is a highly wall-resolved LES [1,7] consisting of a block-structured curvilinear grid with about $12.4 \times 10^{6}$ control volumes using the finite--volume code $\mathcal{L E S O C C}$ (see Section 3.1). The grid points were clustered in the vicinity of the lower wall, the upper wall, and the region where the free shear layer appears. The resolution of the near-wall region can be described by the distribution of non-dimensional $y^{+}$values defined by $y^{+}=\Delta y_{c c} \cdot u_{\tau} / \nu$ where $\Delta y_{c c}$ denotes the distance of the cell center from the wall. The values were below 0.45 with a mean value of about 0.2 except at the windward side of the hill. Here the largest values of the 
wall shear stress were observed and the $y^{+}$value reaches its maximum of about 1.2 . Hence the lower wall is well resolved. Regarding the wall-normal resolution the grid satisfies the requirements of a wall-resolved LES prediction. Compared to previous studies $[15,28]$ who employed in their highly resolved simulations a curvilinear grid with about 4.6 million CVs especially the number of grid points in the wall-normal direction was increased in our reference solution. Furthermore, the simulations resolve not only the lower wall (the hills) in more detail but also resolve the upper wall by a DNS-like representation $\left(y^{+} \leq 0.95\right)$. Thus in contrast to $[15,28]$ the application of a wall function is avoided. Overall the grid is much finer than in [15, 28]. For example, the cell sizes at the hill crest, which is a key region for the periodic hill flow are in the current case $\Delta x_{\text {crest }} / h=0.026$ and $\Delta y_{\text {crest }} / h=2.0 \cdot 10^{-3}$ whereas the corresponding values in $[15,28]$ are $\Delta x_{\text {crest }} / h=0.032$ and $\Delta y_{\text {crest }} / h=3.3 \cdot 10^{-3}$, respectively. Owing to the increased resolution in streamwise and spanwise directions the cell sizes expressed in wall units are below $\Delta x^{+}=20$ and $\Delta z^{+}=9$ and thus lower than in [15,28] and substantially lower than the recommendations for wall-resolved LES given by Piomelli and Chasnov [20]. That also holds at the windward slope of the hill where the largest shear stresses are found. In the reference simulation the dynamic eddy-viscosity model was applied. The boundary conditions were as follows: no-slip conditions at the walls and periodic boundary conditions in spanwise and streamwise directions (fixed mass flow rate). To gather reliable statistical data the flow was averaged in time (dimensionless averaging time $T_{a v g}=1277.34$ ) and along the homogeneous spanwise direction.

In the present investigation simulations for the hill configuration are performed on 4 different grids. Grid A

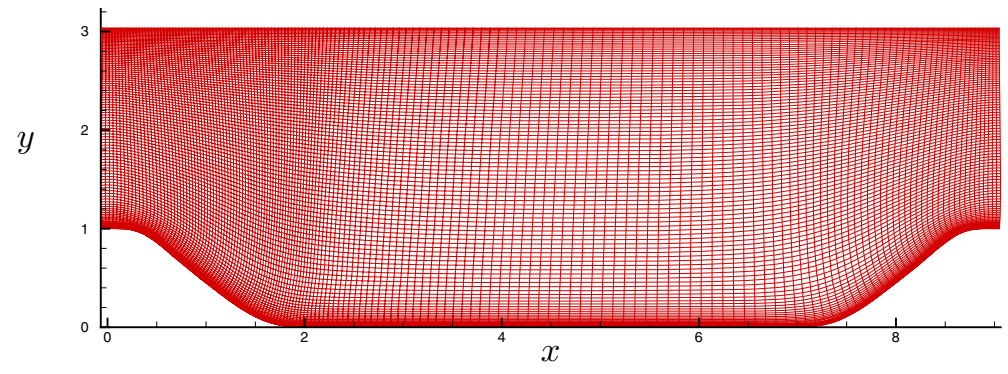

Figure 7: Periodic hill flow test case. Grid B.

$(196 \times 73 \times 100)$ is designed for coarse LES simulation. The height of the first grid cell on the crest of the hill is $\Delta y_{\text {crest }} / h=0.01$. Grid B (Fig. 7) consists of $160 \times 100 \times 60$ control volumes in streamwise, wall-normal (res.: $\Delta y_{\text {crest }} / h=0.005,1^{\text {st }} \mathrm{CV}$ height) and spanwise directions, respectively. It has to be mentioned that grid B has been especially designed for DES. The number of grid points in the spanwise direction is reduced compared with grid A so that the RANS region becomes larger. In this configuration the DES technique using the Spalart-Allmaras model switches from RANS to LES at a distance of about 7-9 RANS cells instead of 4-5 RANS cells for grid A. To study the grid dependency of the DES simulations, a third grid has been used (grid C: $80 \times 100 \times 60$ ), extracted from grid B by retaining every second grid point in the streamwise direction. The RANS region in the DES context for this grid covers 12-14 control volumes away from the wall. Finally, grid D has been designed by using the proposed adaptive grid refinement method applied on grid C (see Section 5.4). Table 1 summarizes the most important characteristics of all simulations carried out for the hill flow test case.

\section{Results}

The hybrid LES-RANS simulations presented in the following are performed with $C_{\text {switch }}$ set to 60 for $y^{*}$ and $y_{2}^{*}$ according to the version used. 


\begin{tabular}{|c||c|c|c|c|c|c|c|}
\hline Grid & Code & Sim. & Model & $N_{x} \times N_{y} \times N_{z}$ & $\Delta x_{\max }^{+}$ & $\Delta z_{\text {max }}^{+}$ & $y_{1^{\text {st } p t, m a x}}^{+}$ \\
\hline Ref. & $\mathcal{L}$ & LES & Dyn. & $280 \times 220 \times 200$ & 20 & 9 & 0.45 \\
\hline \hline A & I & LES & WALE & $196 \times 73 \times 100$ & 40 & 32 & 3.3 \\
\hline & I & DES & SA & $196 \times 73 \times 100$ & 40 & 32 & 3.3 \\
\hline \hline & $\mathcal{L}$ & LES & Smag. & $160 \times 100 \times 60$ & 30 & 30 & 1.0 \\
\hline & $\mathcal{L}$ & Hyb. & Amsch & $160 \times 100 \times 60$ & 35 & 55 & 1.8 \\
\hline & $\mathcal{L}$ & Hyb. & Amsag & $160 \times 100 \times 60$ & 35 & 55 & 1.8 \\
\hline B & $\mathcal{L}$ & Hyb. & Bmsch & $160 \times 100 \times 60$ & 35 & 46 & 1.5 \\
\hline & $\mathcal{L}$ & Hyb. & Bmsag & $160 \times 100 \times 60$ & 35 & 46 & 1.5 \\
\hline & $\mathcal{L}$ & DES & SA & $160 \times 100 \times 60$ & 35 & 33 & 1.1 \\
\hline & I & DES & SA & $160 \times 100 \times 60$ & 35 & 33 & 1.1 \\
\hline \hline C & I & DES & SA & $80 \times 100 \times 60$ & 80 & 38 & 1.2 \\
\hline \hline D & I & DES & SA & $\sim 580,000$ CVs & 50 & 33 & 1.1 \\
\hline
\end{tabular}

Table 1: Hill flow grid resolutions for each simulations. Abbreviations: Ref.: Reference. $\mathcal{L}: \mathcal{L E S O C C}$. I: ISIS. Smag.: Smagorinsky SGS model. Dyn.: Dynamic Smagorinsky SGS model. WALE: Wale SGS model. SA: SpalartAllmaras model. $N_{x}, N_{y}, N_{z}$ : Number of control volumes in streamwise, wall-normal and spanwise directions, respectively. $\Delta x_{\max }^{+}, \Delta z_{\max }^{+}$and $y_{1 \text { st } p t, \max }^{+}$are given for the range $x / h=0$ to 8.0. At the windward side of the hill the resolution decreases owing to the increased value of $u_{\tau}$ in this region.

\subsection{Hybrid LES-RANS technique: Channel flow}

\subsubsection{Versions $A$ and $B$.}

For the classical plane channel flow test case $\left(R e_{b}=10,935\right)$ the new hybrid model provides encouraging statistical results for a coarse $(64 \times 64 \times 64$ control volumes $)$ and a fine grid $(128 \times 128 \times 128$ control volumes $)$. For these resolutions the first grid point (half first cell) is located at position $y^{+}=1.46$ and $y^{+}=0.68$ for the coarse and fine grids, respectively. The results presented and discussed concern the fine grid only. The results on the coarse resolution can be found in [2].

The switching location of the LES-RANS interface is located at $y^{+} \approx 30$ for hybrid version $B$, which is the position expected and estimated based on DNS results. As mentioned in Section 1.3, hybrid version A does not give a precise interface location.

Compared with the DNS data a higher $u_{\tau}$ value is found in the case of the hybrid LES-RANS version B (deviation of $+3.4 \%$ ) whereas hybrid version $A$ predicts a lower $u_{\tau}$ value (deviation of $-2.3 \%$ ). The streamwise velocity of both hybrid versions shows discrepancies in comparison with the DNS reference (see Fig. 8) starting from the core of the RANS region. Furthermore, $U$ presents an inflection point at the location of the interface $\left(y^{+} \approx 30\right)$. This behavior is also observed on the $\overline{u^{\prime} u^{\prime}}$ res curve. This can be explained by the sudden drop of $\nu_{t, R A N S}^{\text {mod }}$ which passes from a RANS to a LES amplitude at the interface (see Fig. 5). Nevertheless, the slope of $U$ in the LES zone is recovered with respect to DNS. Another issue is the over-estimation of $k_{t o t}$. Both hybrid versions show a similar result despite a slightly lower prediction of $k_{\text {mod }}$ by hybrid version B. Thus the loss of modeled turbulent kinetic energy is over-compensated by a gain of resolved energy. As mentioned in Section 1.4.2 the resolved scales do not vanish rapidly at the LES-RANS interface and in the RANS region. The consequence is a high proportion of the resolved field also in the vicinity of the wall (RANS model). Morever, these results show the influence of the interface criterion. It should be kept in mind that the difference between hybrid versions $A$ and $B$ is found in the switching criterion only, whereas the formulation of the RANS model is the same in both cases. The effect of the interface position on the resolved field is seen in Fig. 8 by noticing the lower proportion of $\left(\overline{u_{i}^{\prime} u_{j}^{\prime}}\right)_{\text {res }}$ in hybrid version $A$ compared with version $B$. If version $A$ does not switch 

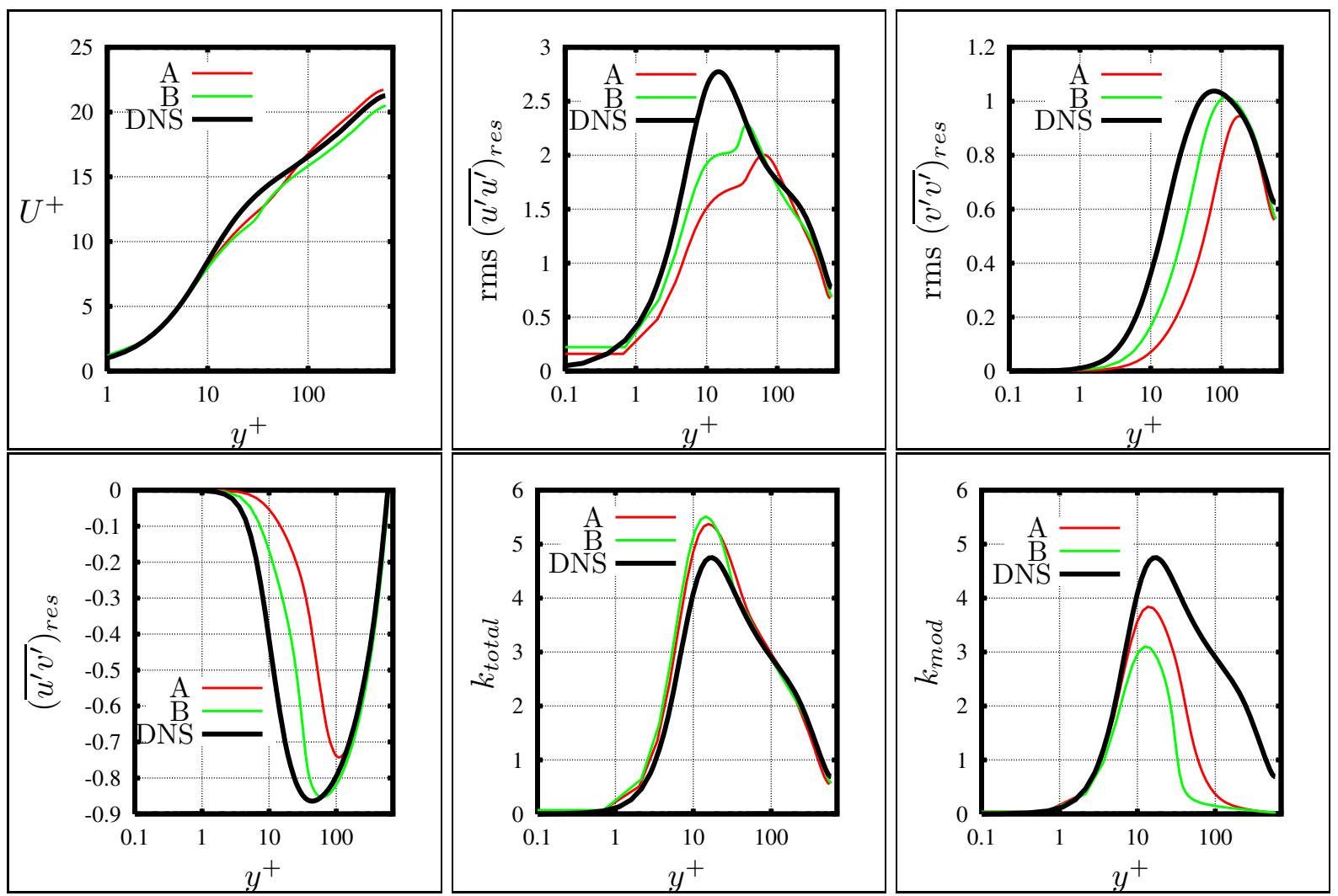

Figure 8: Result for the channel flow test case $(\operatorname{Re}=10,935)$ of hybrid versions $A$ and $B$. Mean streamwise velocity $U^{+}$ (upper left). Resolved Reynolds stresses $\overline{u^{\prime} u^{\prime}}$ (upper center) and $\overline{v^{\prime} v^{\prime}}$ (upper right). Resolved Reynolds shear stress $\overline{u^{\prime} v^{\prime}}$ (lower left). Turbulent kinetic energies $k_{t o t}$ (lower center) and $k_{\text {mod }}$ (lower right). Values scaled with $u_{\tau}$ of each case, respectively. DNS data provided by Moser et al. [18].

from RANS to LES at a clear position, however, it is clear that it offers a more extended RANS region. The consequence is the larger proportion given to the modeled scales in version $A$.

\subsubsection{Versions $A m$ and Bm.}

The first modification carried out on the near-wall model (see Section 1.4.1) only marginally modifies the resolved field as visible in Fig. 9 (center). However, the total turbulent kinetic energy given by the DNS is now predicted accurately by hybrid versions $A m$ and $B m$. Since the results of these two versions are similar, only hybrid versions $B$ and Bm are compared in Fig. 9. Obviously, the adjustment of the RANS model leads to a reduction of the modeled turbulent kinetic energy. However, as previously mentioned, $k_{r e s}$ is kept nearly unchanged. The $u_{\tau}$ value for the versions $A m$ and $B m$ are unchanged compared to the versions $A$ and $B$, respectively. The same remark is valid for the prediction of the mean streamwise velocity.

\subsubsection{Versions Bmf with the compensating function $f\left(y_{2}^{*}\right)$.}

The function designed to compensate the high level of resolved shear stress in the RANS region (see Section 1.4.2) has been applied to the previous hybrid version Bm simulation. The results can been seen in Fig. 10. As can be noticed the mean velocity profile is significantly improved by using the function $f\left(y_{2}^{*}\right)$. A slight deviation can still be observed around the interface. For this purpose the behavior of the model at the interface 

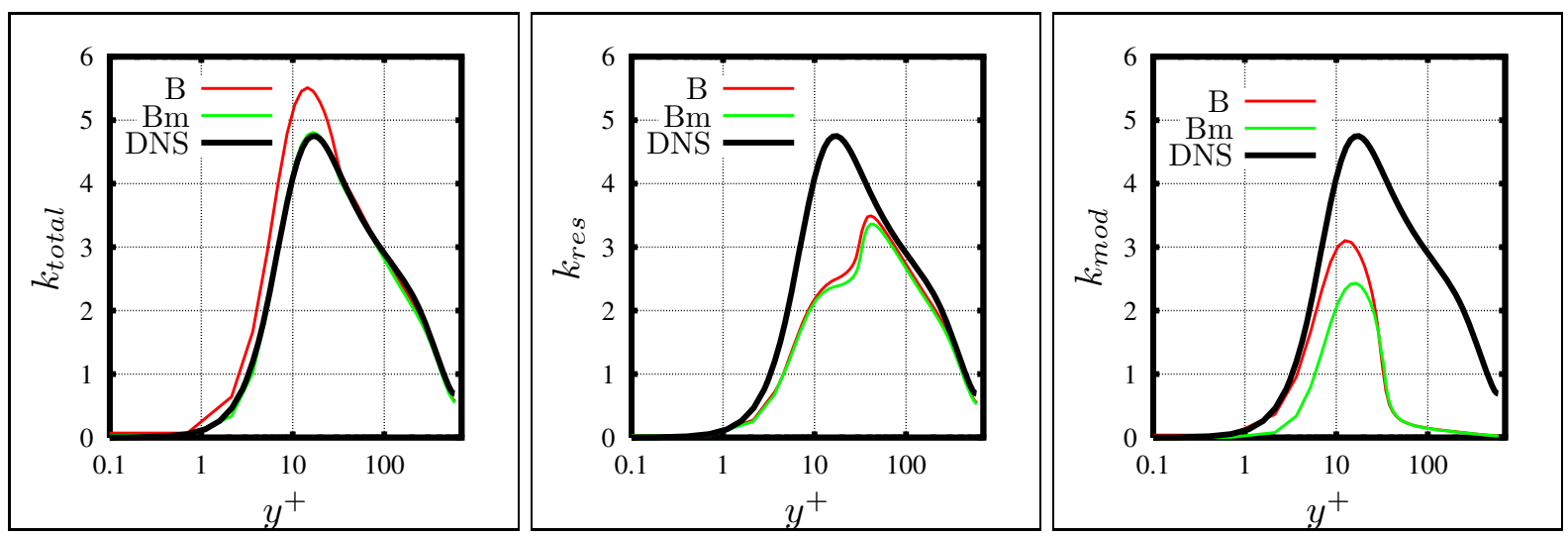

Figure 9: Result for the channel flow test case $(\mathrm{Re}=10,935)$. Turbulent kinetic energies $k_{\text {tot }}$ (left), $k_{\text {res }}$ (center) and $k_{\text {mod }}$ (right) for hybrid versions $B$ and Bm. LES-RANS interface at $y^{+} \approx 30$. Values scaled by $u_{\tau}^{2}$ of each case, respectively. DNS data provided by Moser et al. [18].

is presently studied in order to propose a solution to resolve this discrepancy. In relation to the previous observation for $U^{+}$the prediction of the wall shear stress is in really good agreement with the DNS value $(+0.26 \%$ for $B m f$ compared with $+3.22 \%$ for $B m$ ). The resolved turbulent kinetic energy is increased in the RANS region which is not balanced by the reduction of the modeled part resulting in an over-predicted total kinetic energy $k_{t o t}$.

The function $f\left(y_{2}^{*}\right)$ is presently used to adjust $\nu_{t, R A N S}^{\text {mod }}$ according to the proportion of $\left(\overline{u^{\prime} v^{\prime}}\right)_{\text {res }}$ in the RANS region. This leads to a significant reduction of the $\nu_{t, R A N S}^{\text {mod }}$ value. The maximal instantaneous value of the ratio $\nu_{t, R A N S}^{\text {mod }} / \nu$ is 7.6, 2.7 and 1.007 for the hybrid versions Bm and Bmf and the LES using the one-equation SGS model with Schumann's constants, respectively. As can be noticed the function $f\left(y_{2}^{*}\right)$ brings the hybrid LES-RANS method too close to an LES level, which is not desired. Nevertheless, this type of function will be tested with the hybrid version Am, which offers a lower level of resolved contributions and a higher $\nu_{t, R A N S}^{\text {mod }}$ value. Thus the resulting hybrid version Amf should provide a suitable $\nu_{t, R A N S}^{\text {mod }}$ value.

\subsection{Hybrid LES-RANS technique: Periodic hill flow simulation}

The second, reasonably complex test case is the flow over periodic hills $\left(R e_{b}=10,595\right)$. To assess the new hybrid approach, grid B is used (depicted in Fig. 7). Whereas the previous hybrid LES-RANS results shown for the channel flow test case were performed with the SGS model of Schumann (see Section 1.1) in LES mode, in the following the hybrid LES-RANS versions Am and Bm are also assessed with the SGS model of Sagaut [24] using $C_{\mu}=0.062$ and $C_{d}=1.0$ as constants. These tests are performed in order to check the influence of the SGS model. The different variants are named hybrid LES-RANS versions Amsch and Bmsch and Amsag and Bmsag for the SGS model of Schumann and Sagaut, respectively (see Table 1). Additionally, an LES using the Smagorinsky model has been performed.

The RANS and LES domains are shown in Fig. 11. The near-wall region is totally treated in RANS mode. For the hybrid versions Bm (Bmsch and Bmsag), the interface position occurs at a distance of 5-10 RANS cells from the wall (depending on the streamwise location). The separation region contains 10 RANS cells whereas the hill crest is covered by 5 cells. The interface at the lower wall is located above $6-12$ RANS cells for the hybrid versions Am (Amsch and Amsag). It is of interest to note that in this case these versions do not show a problem of interface distinction as critical as encountered in the channel flow case.

The hill flow results (see Fig. 12) of the different variants of the hybrid model are plotted together with DES [27] results using the Spalart-Allmaras model [26] and the highly-resolved reference LES data. The mean velocity $U$ 

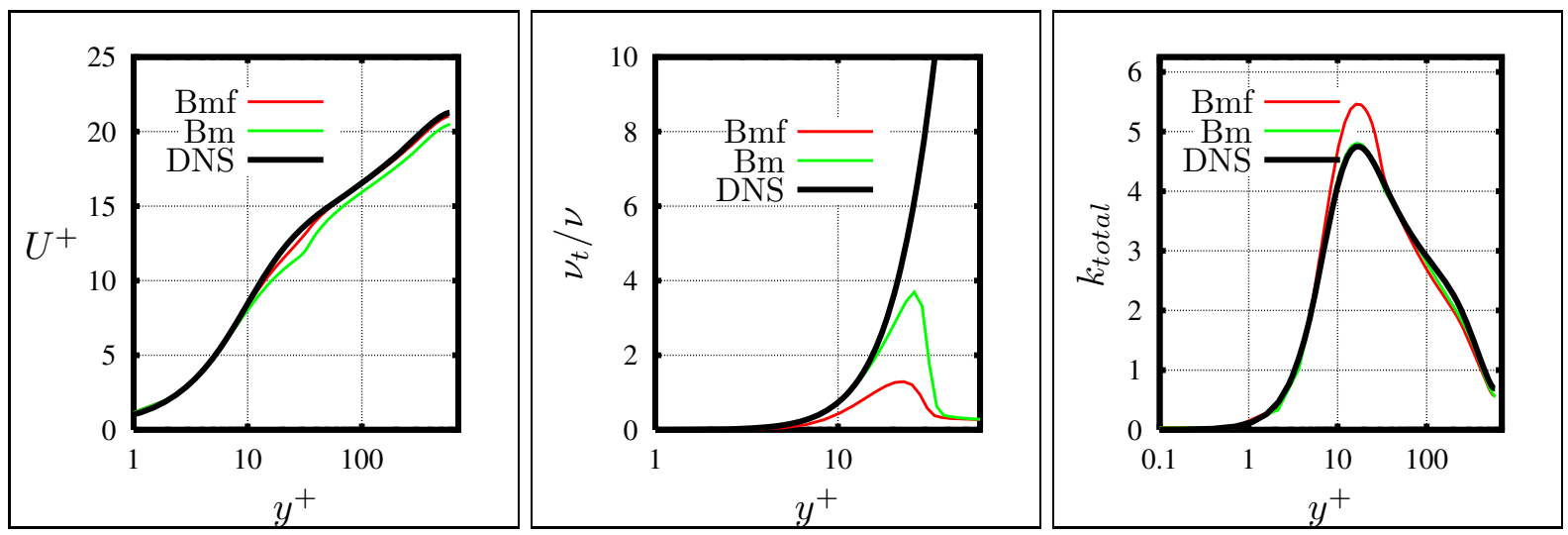

Figure 10: Channel flow test case $(\mathrm{Re}=10,935)$. Results for hybrid versions $B m$ and Bmf. Mean streamwise velocity $U^{+}$(left). Averaged modeled turbulent viscosity ratio $\nu_{t, R A N S}^{\text {mod }} / \nu$ (center). Total turbulent kinetic energy $k_{\text {tot }}$ (right). LES-RANS interface at $y^{+} \approx 30$. DNS data provided by Moser et al. [18].

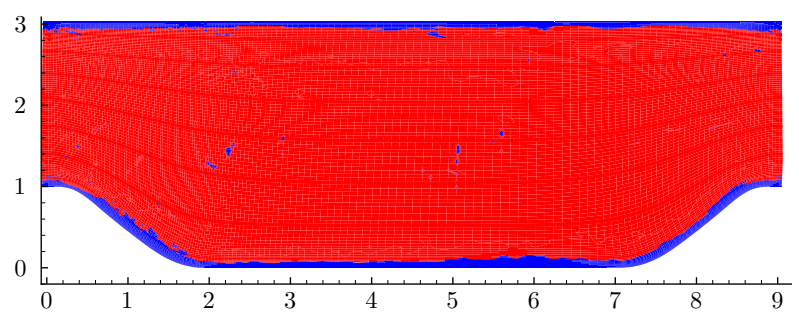

(a) Hybrid version Amsch

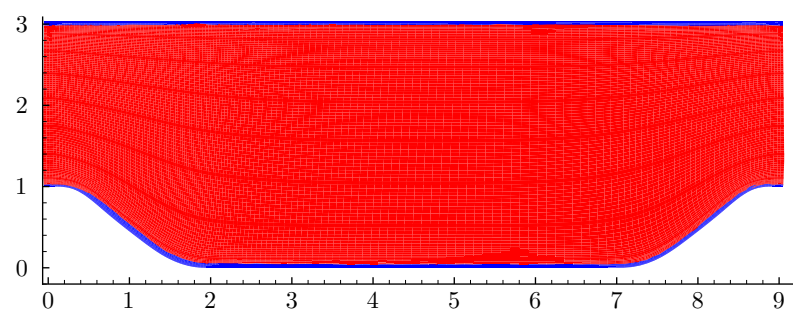

(b) Hybrid version Bmsch

Figure 11: Results for the periodic hill flow at $R e_{b}=10,595$. LES-RANS domain layout for hybrid versions Amsch (left) and Bmsch (right). RANS domain: blue zone. LES domain: red zone.

normalized with the bulk velocity is plotted at the positions $x / h=0.5,2$ and 6 , which represent the beginning and the center of the recirculation region and the flow after reattachment, respectively. $U$ is well reproduced by the new models at each streamwise positions. However, concerning the wall shear stress distribution $\tau_{w}$ the hybrid simulations show at the hill crest larger deviations than the DES, which is in good agreement with the reference LES. This behavior was also noticed in RANS simulations of the hill flow test case. Regarding the recirculation region the LES applying the Smagorinsky model predicts the best agreement regarding the location of the separation point with respect to the reference LES (see Table 2). Whereas the DES under-predicts the separation location but stays in good agreement with the reference, the hybrid simulations delay this point. However, both hybrid versions Amsch and Amsag seem to give slightly better results. All hybrid and DES simulations show a delayed reattachment compared to the reference solution. Marginally, the hybrid versions Bmsch and Bmsag are in closer agreement with the reference data than the DES result. More interesting is that the hybrid versions Amsch and Amsag show better predictions of the reattachment location than the DES and the LES. Even if the hybrid Amsag slightly over-predicts the reattachment point with respect to version Amsch, it still offers a better prediction than the LES. The hybrid version Amsch presents the most accurate reattachment point. In comparison with LES and DES, which show deviations of $-3.1 \%$ and $+10.7 \%$, respectively, version Amsch leads to a deviation of $+1.2 \%$ only. Fig. 13 shows plots of the averaged streamlines for hybrid versions Amsch and Amsag, DES using the Spalart-Allmaras model, and the LES reference solution. Both hybrid and 
DES techniques exhibit slightly larger recirculation regions with respect to the LES reference. The center of this reciculation zone is predicted similarly by the hybrid and DES simulations. However, in comparison with the reference data this location is slightly shifted backward. Nevertheless, these three simulations, hybrid versions Amsch, Amsag and DES still offer satisfactory results.

In general, the Reynolds stresses are well predicted by all hybrid variants (see Fig. 12). The profiles are overall recovered with respect to the reference LES. Regarding the intensity, only slight under- or over-estimations are observed. However, at the positions $x / h=0.05$ and 0.5 the versions Amsch and Amsag show discrepancies (under-estimation) in the prediction of the peaks located at the vicinity of the lower wall for $\overline{v^{\prime} v^{\prime}}$ tot and $\overline{u^{\prime} v^{\prime}}$ tot . The modeled stresses are similar in all four simulations, thus these under-estimations result from a lack of resolved scales in this region for the variants Amsch and Amsag compared with the versions Bmsch and Bmsag. Nevertheless, these discrepancies are not observed in the versions Bmsch and Bmsag, for which a satisfactory good agreement is obtained at each positions and for each Reynolds stresses. More results can be found in [2,3]. In conclusion, all hybrid simulations give encouraging statistical results similar (or in some cases better) than DES. However, the hybrid versions Amsch and Amsag can be seen as superior in the prediction of the recirculation region. Oppositely, the hybrid versions Bmsch and Bmsag show better predictions of high-order statistics than the versions Amsch and Amsag. Concerning the constants of the one-equation SGS model in the hybrid simulations, the previous results shows a slight advantage of the model of Schumann.
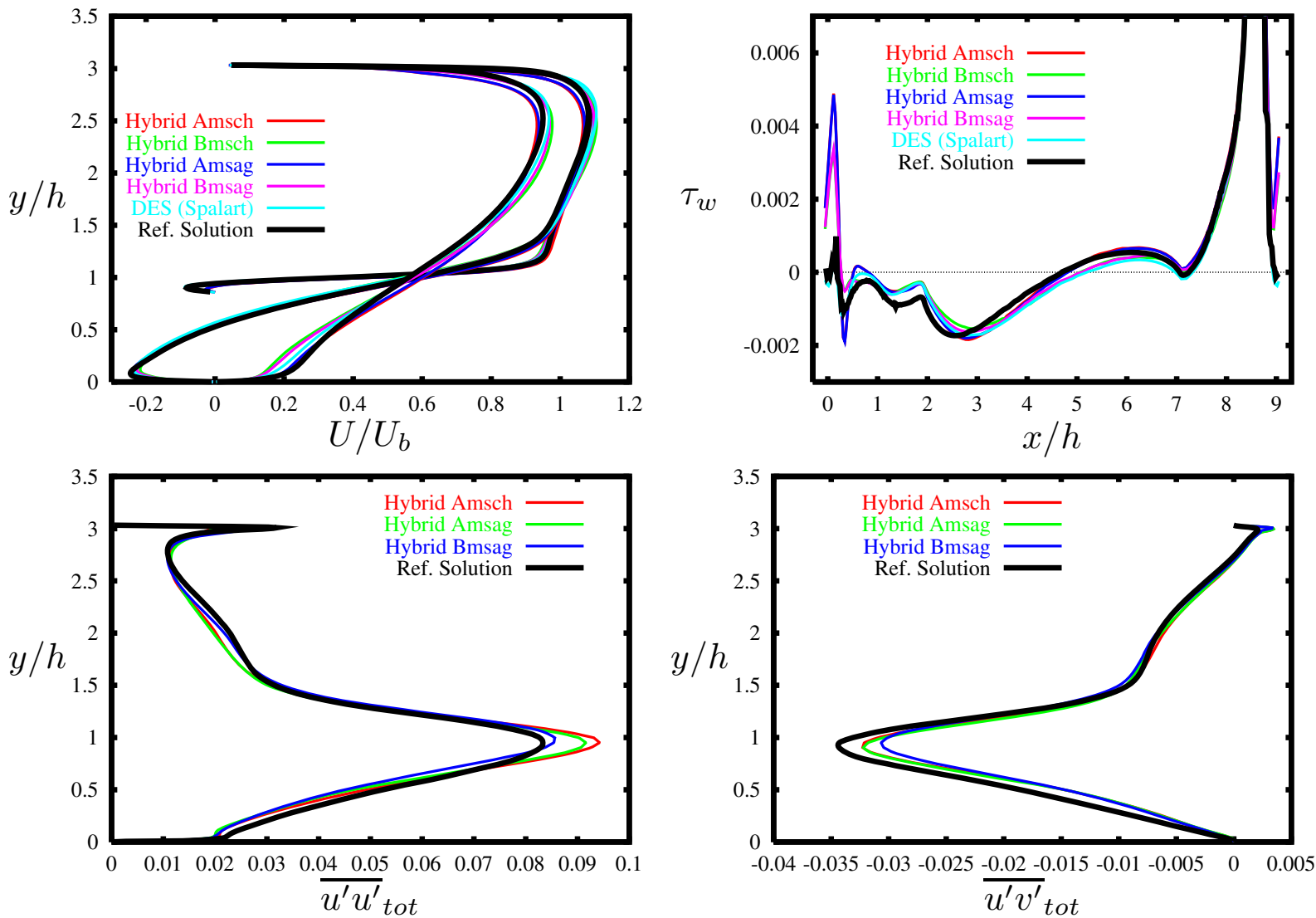

Figure 12: Results for the periodic hill flow at $R e_{b}=10,595$. Mean velocity $U / U_{b}$ at positions $x / h=0.5,2$ and 6 (upper left). Wall shear stress $\tau_{w}$ at the lower wall (upper right). $\overline{u^{\prime} u^{\prime}}$ tot (lower left) and ${\overline{u^{\prime} v^{\prime}}}_{\text {tot }}$ (lower right) at position $x / h=2$. 


\begin{tabular}{|c||c|c||c|c|}
\hline Name & Sep. loc. & Diff. with ref. & Reat. loc. & Diff. with ref. \\
\hline Reference $\boldsymbol{L} \boldsymbol{E}$ S & 0.190 & - & 4.694 & - \\
\hline LES Smagorinsky & 0.201 & $+5.79 \%$ & 4.547 & $-3.1 \%$ \\
\hline DES SA model & 0.173 & $-8.95 \%$ & 5.197 & $+10.7 \%$ \\
\hline Hybrid Amsch & 0.254 & $+33.7 \%$ & 4.751 & $+1.2 \%$ \\
\hline Hybrid Bmsch & 0.268 & $+41.05 \%$ & 5.065 & $+7.9 \%$ \\
\hline Hybrid Amsag & 0.255 & $+34.2 \%$ & 4.812 & $+2.5 \%$ \\
\hline Hybrid Bmsag & 0.271 & $+42.6 \%$ & 5.096 & $+8.6 \%$ \\
\hline
\end{tabular}

Table 2: Results for the periodic hill flow at $R e_{b}=10,595$. Separation and reattachment locations and differences observed with respect to the reference solution $[1,7]$.

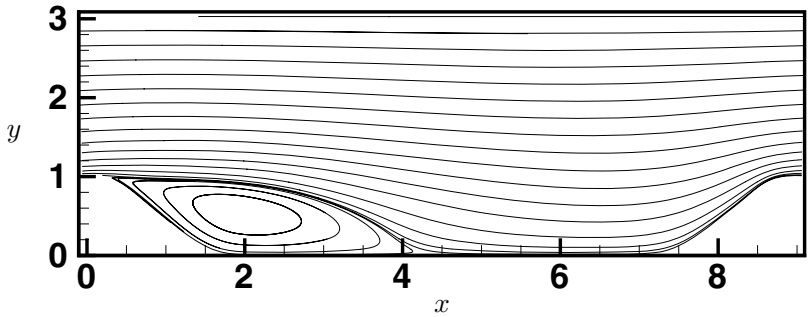

(a) LES Ref. solution

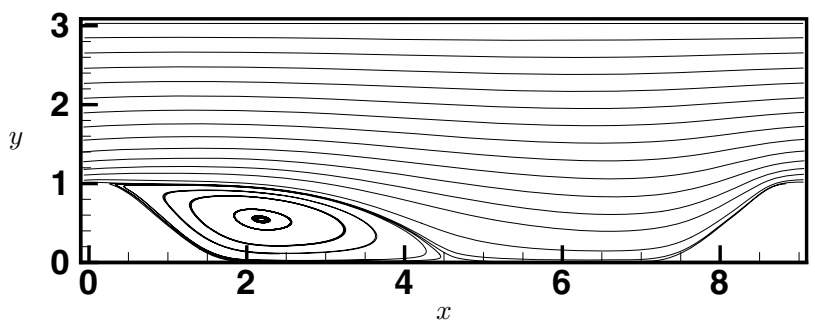

(c) DES SA model

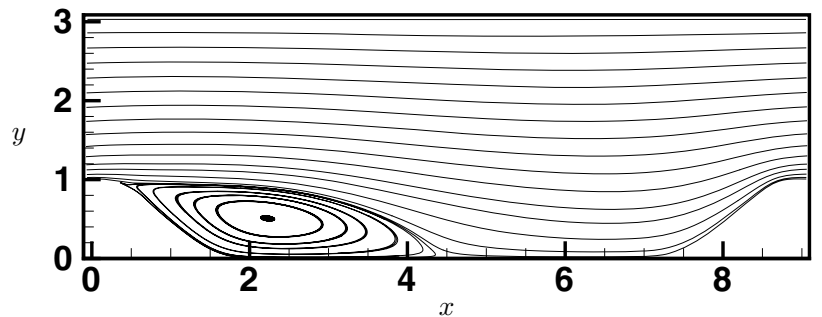

(b) Hybrid version Amsch

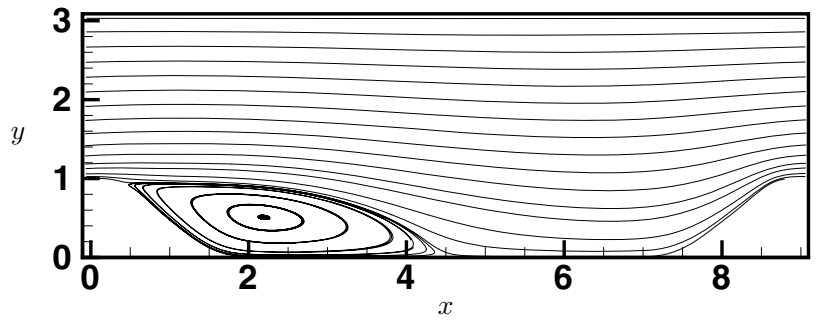

(d) Hybrid version Amsag

Figure 13: Results for the periodic hill flow at $R e_{b}=10,595$. Streamlines of the averaged flow.

\subsection{DES simulations including a comparison of codes}

Concerning LES, simulations have been performed on grids A and B with two subgrid scale models in order to investigate the effect of numerical error and subgrid-scale modeling. Both codes were applied, $\mathcal{L} \mathcal{E S O C C}$ with the Smagorinsky model and ISIS with the WALE model [13,19]. Results and comparisons between both codes as well as additional validated codes concerning the hill flow test case in the LES context are available in $[1,3]$. We focus here on the comparison of DES simulations with the SA model. For ISIS, a second-order central difference scheme is used both for the convection and diffusion terms in the momentum equation. A GDS scheme is employed for the transport equation of the turbulent eddy-viscosity. It behaves as a central difference scheme when the solution is locally monotonic, but switches to a first-order upwind scheme otherwise. The dimensionless time step employed in all simulations is 0.007. Four nonlinear iterations are performed at each time step, which allows to reduce the nonlinear residual by 2 to 3 orders. The dimensionless averaging time is about 1800 and 800 for the simulations performed on grid A and grid B, respectively. For $\mathcal{L} \mathcal{E} \mathcal{S O C C}$, the spatial discretization of all fluxes is based on a central scheme of second-order accuracy. A low-storage multi-stage 

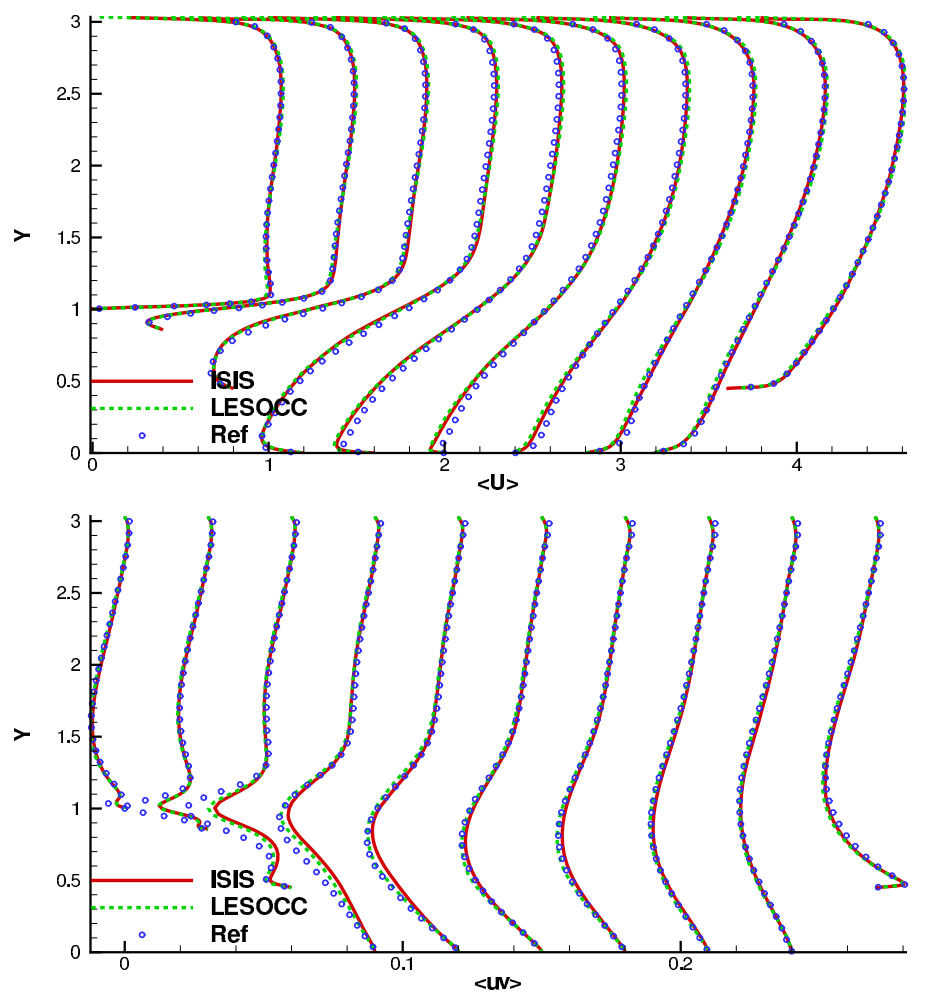

Figure 14: Comparison of DES results. Streamwise velocity component $U / U_{b}$ (upper) and Reynolds shear stress $\overline{u^{\prime} v^{\prime}}$ (lower) of $\mathcal{L E S O C C}$ and ISIS at positions $x / h=0.05,0.5,1,2,3,4,5,6,7$ and 8 .

Runge-Kutta method (second-order accurate) is applied for time-marching. The dimensionless time step and averaging time used for the DES simulation on grid B are 0.004 and about 605 , respectively.

\subsubsection{Comparison of codes.}

The results (streamwise velocity component $U$, Reynolds shear stress $\overline{u^{\prime} v^{\prime}}$ and two normal Reynolds stresses $\overline{u^{\prime} u^{\prime}}$ and $\overline{v^{\prime} v^{\prime}}$ ) of two DES simulations performed on the same grid (grid B) using the same SA model with the ISIS code and the $\mathcal{L E S O C C}$ code are compared.

The streamwise velocity is shown beside $\overline{u^{\prime} v^{\prime}}$ in Fig. 14 whereas the two normal stresses are presented in Fig. 15. Although the prediction for the mean flow agrees well with the reference solution for both codes, some discrepancies are observed concerning the normal stresses. The subgrid eddy-viscosity predicted by both codes is quite similar. The maximum value of the mean subgrid eddy-viscosity is about ten times the molecular viscosity in the region between the two hills (Fig. 16). The minor deviations found between the results of both codes are assumed to be the effect of partially different numerical schemes and implementation aspects.

\subsubsection{Grid dependency issue.}

To investigate the influence of the grid resolution on the results, grid $\mathrm{C}$ was applied and the results of the DES predictions obtained on grid B are used for comparison. Although not shown here, in pure RANS simulation both grids are capable of providing a grid independent solution using the Spalart-Allmaras model [26] at the given Reynolds number. In DES, the solution is not grid independent as can be seen in Fig. 17. The prediction of the normal Reynolds stress $\overline{u^{\prime} u^{\prime}}$ obtained on grid $\mathrm{C}$ is over-predicted, probably because of the coarser grid density in this region. 

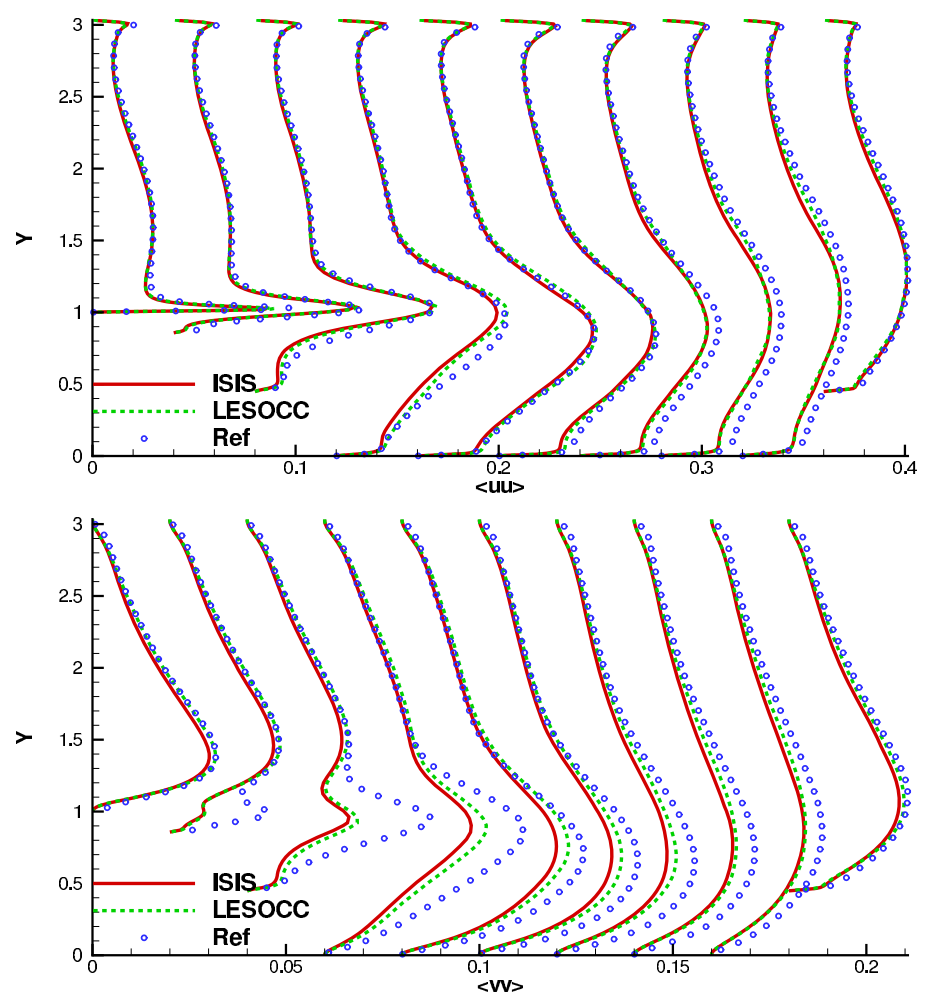

Figure 15: Comparison of DES results. $\overline{u^{\prime} u^{\prime}}$ (upper) and $\overline{v^{\prime} v^{\prime}}$ (lower) of $\mathcal{L E S O C C}$ and ISIS at positions $x / h=$ $0.05,0.5,1,2,3,4,5,6,7$ and 8 .
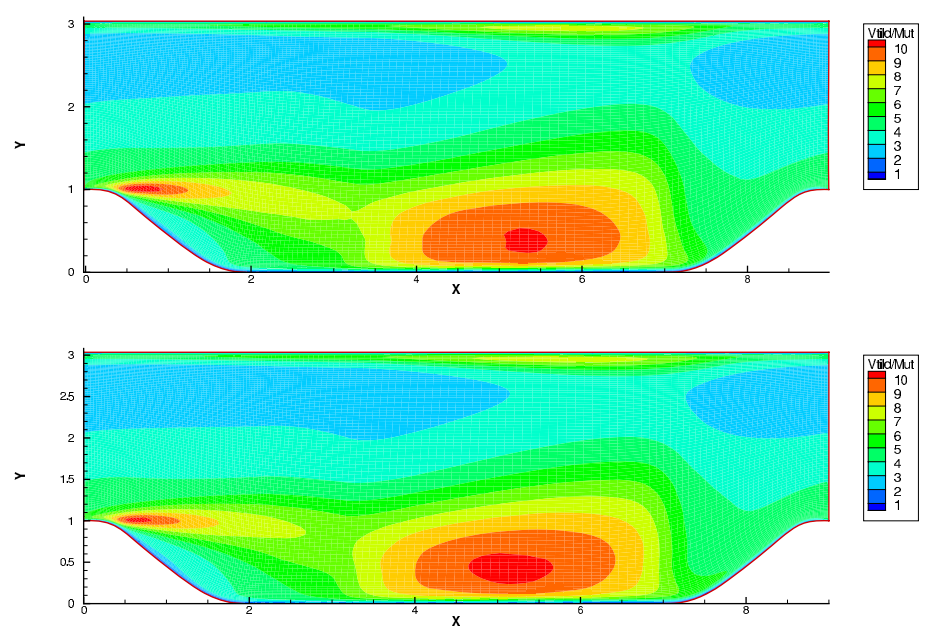

Figure 16: Mean turbulent eddy-viscosity. Upper: $\mathcal{L} \mathcal{E S O C C}$, lower: ISIS. 

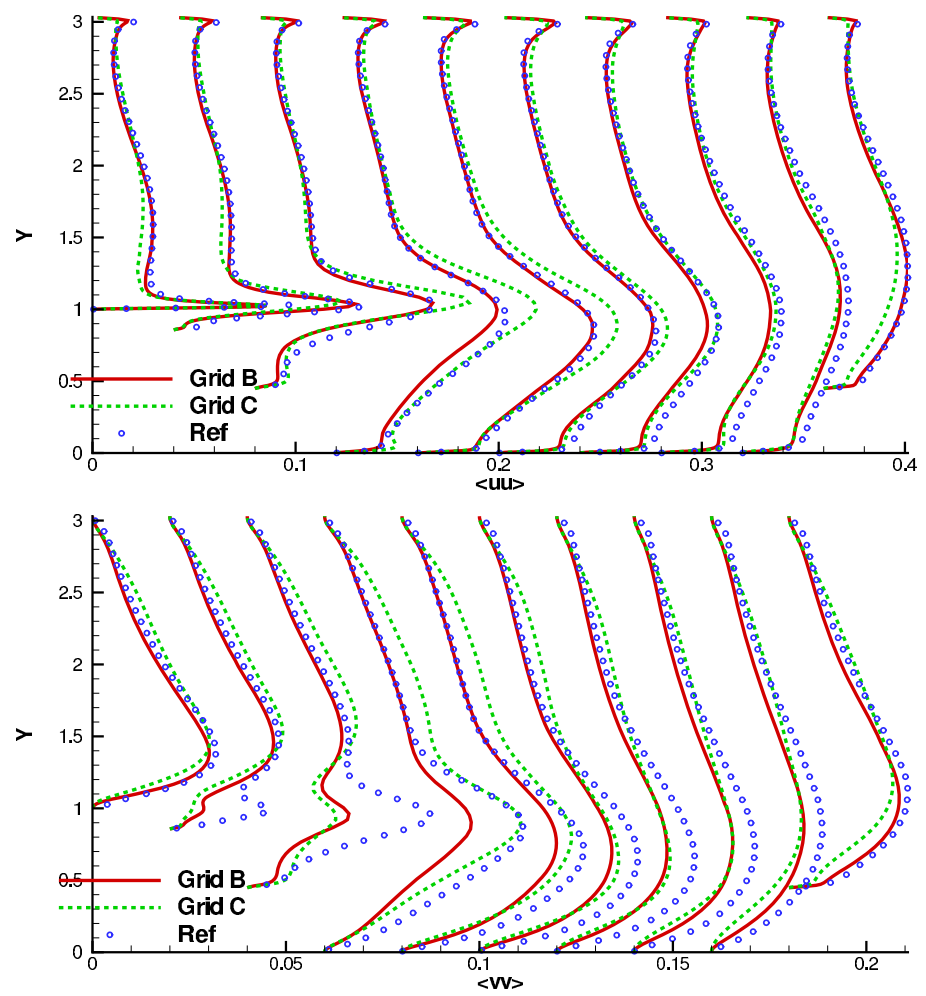

Figure 17: Comparison of $\overline{u^{\prime} u^{\prime}}$ (upper) and $\overline{v^{\prime} v^{\prime}}$ (lower) between the DES simulations performed on grids B and C using ISIS.

Nevertheless, little discrepancies are observed between the mean velocity profiles provided by the simulations on both grids $\mathrm{B}$ and $\mathrm{C}$ as shown in Fig. 18. The grid resolution of grid $\mathrm{C}$ seems to be sufficient to capture the behavior of the large-scale motion of the flow correctly. More results can be found in $[2,3,8,9]$. It is of interest in a near future to compare these computations with simulations performed on coarser grids to investigate the grid dependency issue, which can give useful information for the grid refinement procedure.

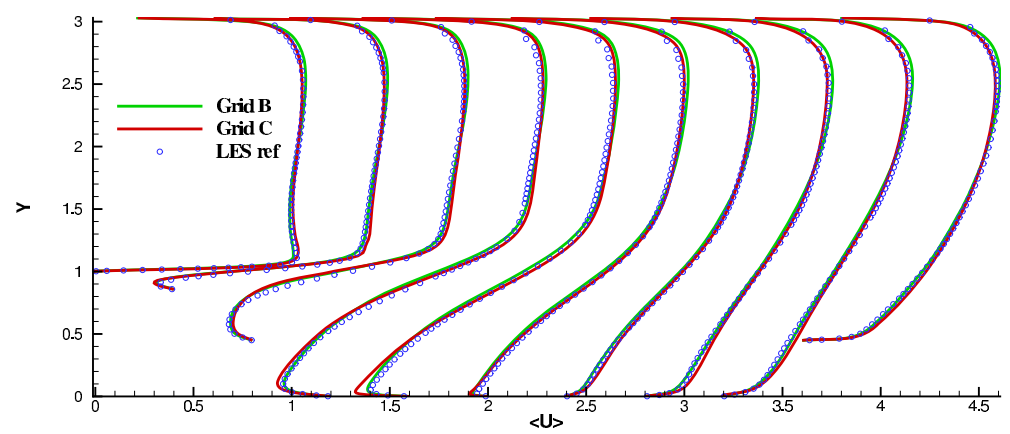

Figure 18: Comparison of mean streamwise velocity profiles between the DES simulations performed on grids B and C using ISIS. 

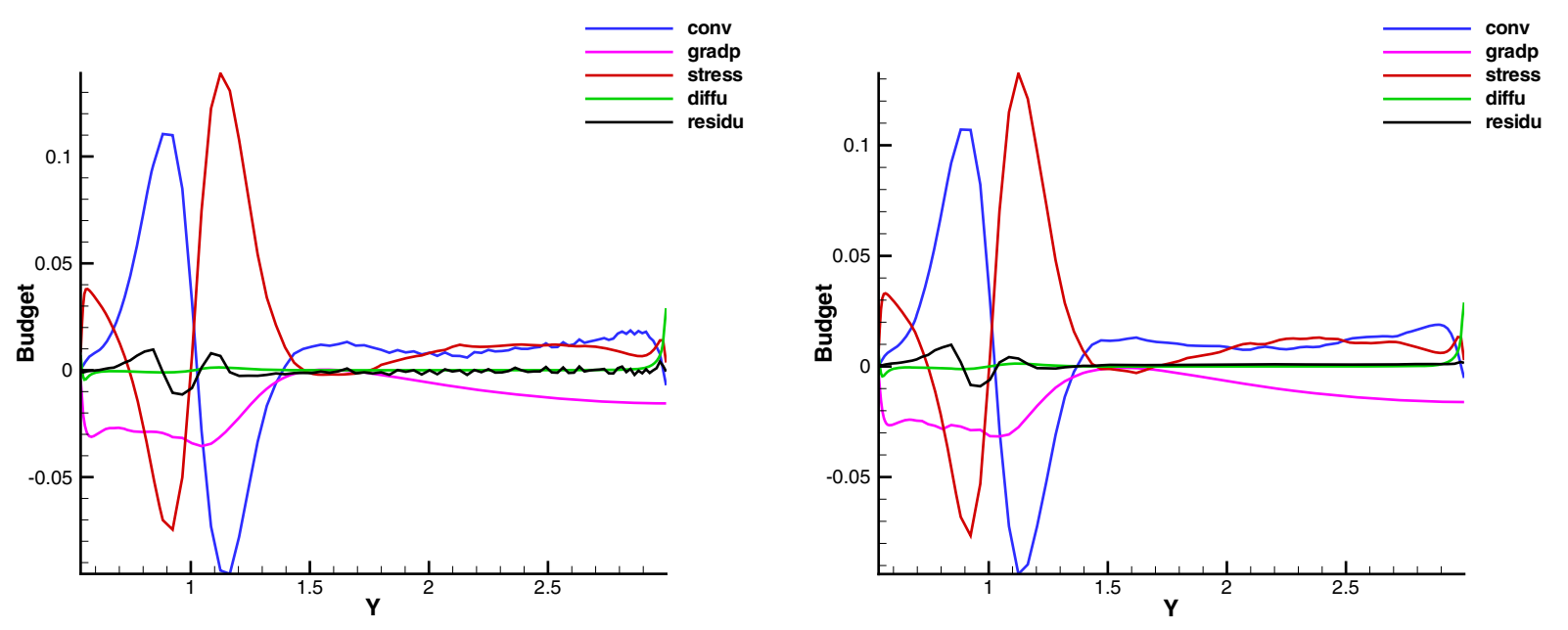

Figure 19: Evaluation of the budget of the $U$-momentum equation for $\mathcal{L E S O C C}$ (left) and ISIS (right) at $x / h=1.05$ on grid B. Abbreviations: conv: Convection term. gradp: Pressure gradient. stress: Modeled and resolved stresses. diffu: Diffusion term. residu: Residual of the budget.

\subsection{Grid refinement}

\subsubsection{Numerical accuracy assessment.}

Unlike DNS and RANS simulations, the spatial resolution in LES simulations is usually insufficient and the numerical error is not trivial to quantify. Nevertheless, it is possible to formulate some indicators which can be used to assess the numerical accuracy. One of such indicators is the balance of the momentum equations for the mean flow. The levels of momentum balance at the location $x / h=1.05$ for grid B are shown in Fig. 19. This balance includes modeled and resolved scales. Even if not shown here, the evaluations of these levels have been performed in the RANS context using the same grid. It seems that the level of the residuals is much higher in DES than in RANS and not negligible with respect to other terms of the budget. In RANS simulations, the regions of high residual level are related to the grid rather than to the flow, whereas in DES the regions of high level of residual are related to both the flow and the grid. To enhance the results, an improvement of the grid density by refinement is required, especially in the free shear layer after separation [8].

Statistics of the mean flow quantities are evaluated at the cell-centered position. Nevertheless, modeled and resolved scales are reconstructed on the faces of the cells to build the budget of the momentum equation. This explains the non-zero value of the momentum budget. The observed high level of residual indicates that the numerical error in DES simulations is not negligible. How to improve the accuracy? In order to answer this question, further investigations have been performed. For that purpose grid B is used, for which attention during its design has been taken to ensure the grid smoothness. Then the results obtained by $\mathcal{L E S O C C}$ and ISIS on grid B using the same DES model are compared. Fig. 20 displays the balance of the $U$-momentum equation for both codes. The mean steamwise pressure gradient is included in the evaluation of the balance in the mean momentum equation shown here. The location of the region of high residual is similar for both simulations. The highest residual is found in the free shear layer after the separation. The grid needs to be refined in this region in order to improve the accuracy of the prediction. The second region of high residual region is located in the separation zone after $x / h=2$. The residual rises in this part due to the fact that the cell size is increased in this particular region. The third high residual region is found near the windward side of the hill. It seems desirable to increase the grid density in this area to represent the high level of fluctuations in 


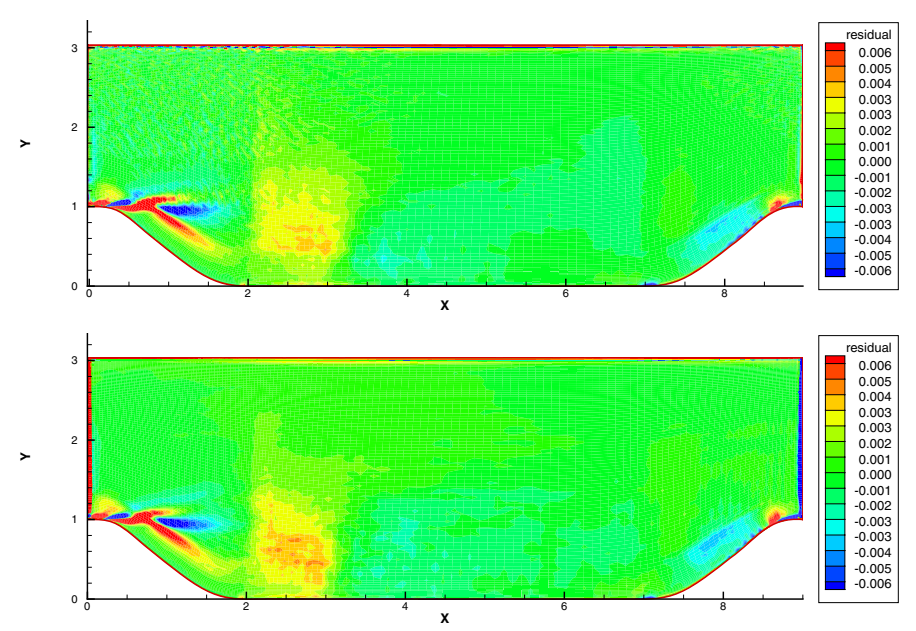

Figure 20: Residual of the balance of the $U$-momentum equation for $\mathcal{L E S O C C}$ (upper) and ISIS (lower) obtained on grid B.

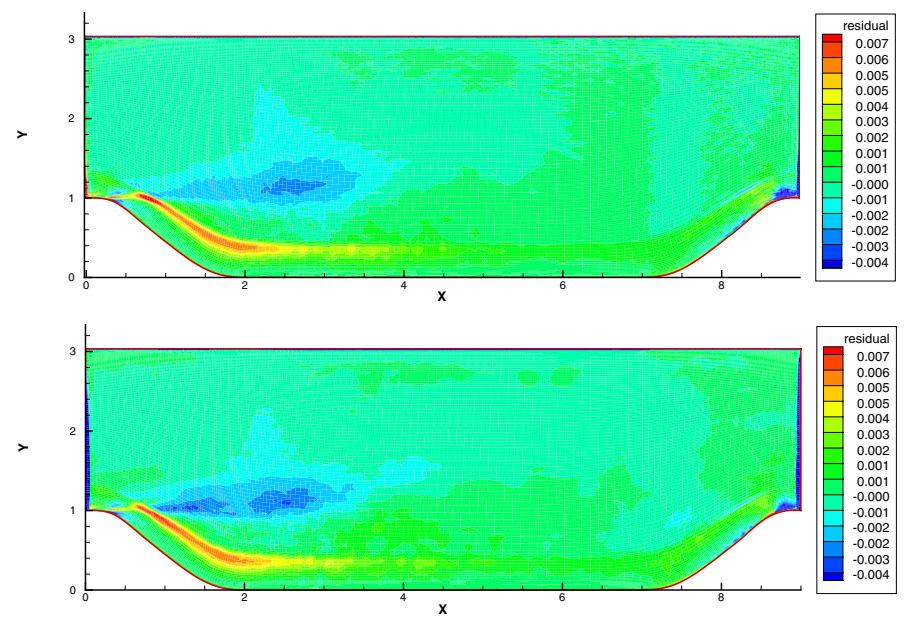

Figure 21: Residual of the balance of the $V$-momentum equation for $\mathcal{L} \mathcal{E S O C C}$ (upper) and ISIS (lower) obtained on grid B.

the spanwise direction due to the impinging flow on the hill.

In conclusion, both ISIS and $\mathcal{L E S O C C}$ show the same regions of high residual value. A similar comparison is performed for the $V$-momentum equation. Both codes show a high residual region due to the grid near the lower wall where the transition from a wall-orthogonal to a non-orthogonal grid generated by different technique occurs (Fig. 21).

The same comparison between the results obtained on grid $\mathrm{B}$ and those related to grid $\mathrm{C}$ shows that the level of this residual increases (Fig. 22). Since the residual error is correlated to the spatial resolution, it can be employed as criterion for grid refinement. Although not shown here, similar observations and analysis were performed upon LES simulations with the WALE model on the same grids [9]. 

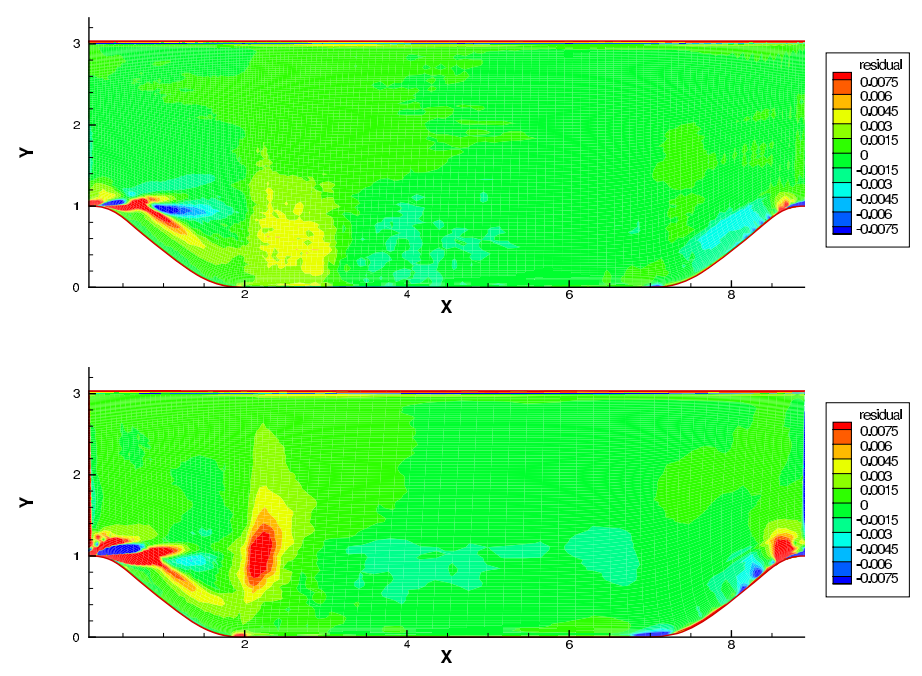

Figure 22: Comparison of the residual of the balance of the $U$-momentum equation obtained on grids B (upper) and C (lower) using ISIS.

\subsubsection{Adaptive grid refinement.}

The analysis performed in the previous section shows that in LES and DES predictions the identification of regions where the numerical error is high, is possible. To reduce this error, two solutions are available. Either the order of accuracy of the spatial discretization scheme is increased or the local grid refinement of these regions is realized. The second solution can be applied within the ISIS code. Fig. 23 shows an example of a refined grid based on grid $\mathrm{C}$ using the adaptive local refinement procedure. This last grid is called grid D. In this procedure the refinement criterion is based on the residual of the $U$ and $V$ mean momentum equations. The procedure of refinement is simple: cells where the evaluation of the level of the residual $(U$ or $V)$ is higher than a fixed limit are refined automatically by dividing them into four new cells. The limit is fixed here so that the number of point of the refined grid remains reasonable.

Since grid C already gives acceptable results as seen in Section 5.3.2, the comparison of results obtained on grids $\mathrm{C}$ and $\mathrm{D}$ does not show a real quality improvement of the solution on the refined grid. Besides, grid D differs from grid C by only a few control volumes (about 580,000 CVs for grid D), which has led to mainly the same solutions on the grids as shown for example for the mean velocity profiles (Fig. 24) and the averaged Reynolds stresses (Fig. 25).

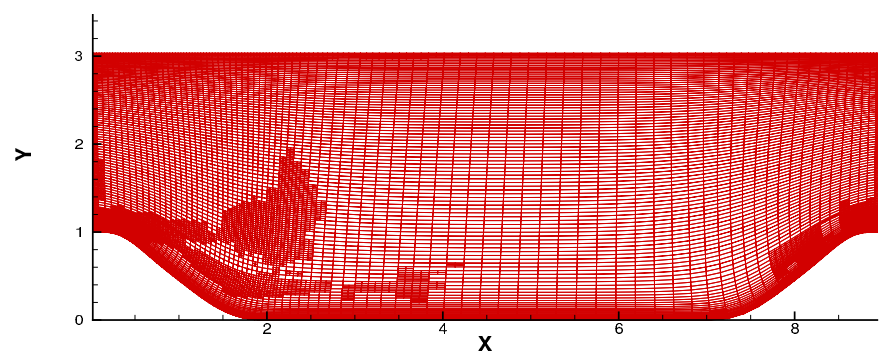

Figure 23: Grid D with adaptive local refinement. 


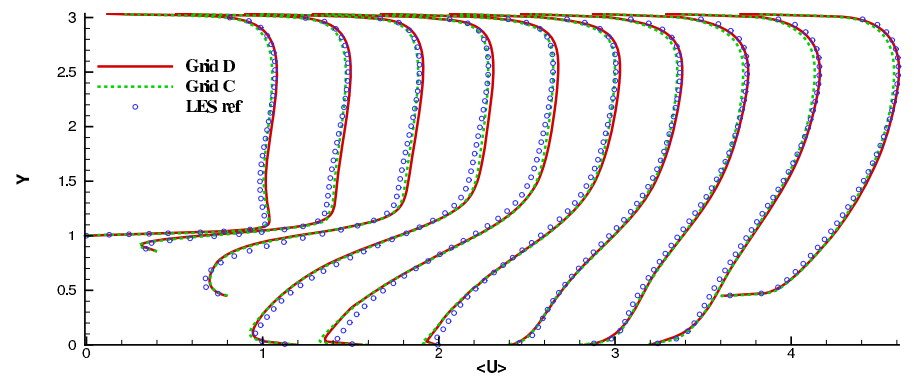

Figure 24: Comparison of mean streamwise velocity profiles obtained on grids C and D using ISIS.

In order to really notice the effects of refinement on the solution, we plan to perform the same procedure on a coarser initial grid than grid $\mathrm{C}$ in future work. To assess the procedure of grid refinement in DES and LES context, other investigations are also under study such as defining new refinement criteria and studying the influence of the subgrid models on the procedure.
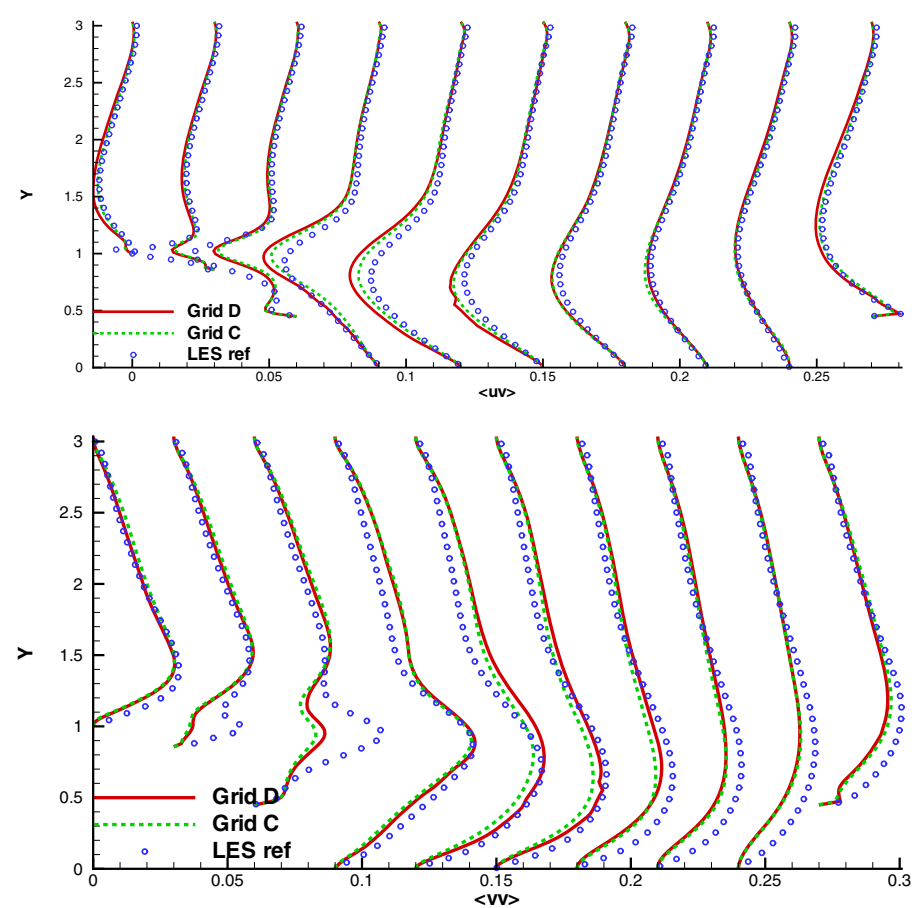

Figure 25: Comparison of $\overline{u^{\prime} v^{\prime}}$ (upper) and $\overline{v^{\prime} v^{\prime}}$ (lower) obtained on grids $\mathrm{C}$ and $\mathrm{D}$ using ISIS.

\section{Conclusions}

This study has focused on the hybrid LES-RANS technique. A new non-zonal hybrid LES-RANS approach which splits up the simulation into a near-wall RANS part and an outer LES part was suggested and tested. 
This technique is based on the association of a one--equation SGS model (LES zone) with the near-wall oneequation model proposed by Rodi et al. (1993) in the RANS zone. First investigations with this new approach reveal encouraging results for both the plane channel flow and the periodic hill flow. Presently, the LES-RANS interface region and appropriate grid-independent switching criteria are investigated in detail and modifications undergone on the RANS and LES models are tested. In the near future new test cases will be used in order to assess the present hybrid LES-RANS technique on different geometries and under different flow conditions.

Furthermore, attempts have been made to improve the accuracy of DES and LES simulations by using adaptive local grid refinement. For this purpose, a criterion based on the residual of the budget of the mean momentum equations has been studied. Currently, the procedure is tested with different structured and unstructured coarser grids to evaluate its influence on the quality of the solutions. Other test cases and subgrid-scale models will be used in order to assess the interest of such refinement criteria in the LES context.

\section{REFERENCES}

[1] Breuer, M., Jaffrézic, B., Peller, N., Manhart, M., Fröhlich, J., Hinterberger, Ch., Rodi, W., Deng, G., Chikhaoui, O., S̆arić, S., Jakirlić, S. (2006) A Comparative Study of the Turbulent Flow Over a Periodic Arrangement of Smoothly Contoured Hills, Sixth Int. ERCOFTAC Workshop on DNS and LES: DLES-6, Poitiers, France, Sept. 12-14, 2005, ERCOFTAC Series, vol. 10, pp. 635-642, Direct and Large-Eddy Simulation VI, eds. E. Lamballais, R. Friedrich, B.J. Geurts, O. Métais, ISBN-10 1-4020-4909-9, Springer Science, (2006).

[2] Breuer, M., Jaffrézic, B. (2005) Hybrid LES-RANS Technique Based on a One-Equation Near-Wall Model, EUROMECH Colloquium 469, Large-Eddy Simulation of Complex Flows, TU Dresden, Germany, Oct. 6-8, 2005.

[3] Breuer, M., Jaffrézic, B., Šarić, S., Jakirlić, S., Deng, G., Chikhaoui, O., Fröhlich, J., von Terzi, D., Manhart, M., Peller, N. (2005) Issues in Hybrid LES-RANS and Coarse Grid LES of Separated Flows, EUROMECH Colloquium 469, Large-Eddy Simulation of Complex Flows, TU Dresden, Germany, Oct. 6-8, 2005.

[4] Breuer, M., Rodi, W. (1996) Large-Eddy Simulation of Complex Turbulent Flows of Practical Interest, In: Flow Simulation with High-Performance Computers II, ed. E.H. Hirschel, Notes on Numerical Fluid Mechanics, vol. 52, pp. 258-274, Vieweg Verlag, Braunschweig.

[5] Breuer, M. (1998) Large Eddy Simulation of the Sub-Critical Flow Past a Circular Cylinder: Numerical and Modeling Aspects, Int. J. for Num. Methods in Fluids, vol. 28, pp. 1281-1302.

[6] Breuer, M. (2002) Direkte Numerische Simulation und Large-Eddy Simulation turbulenter Strömungen auf Hochleistungsrechnern, Habilitationsschrift, Univ. Erlangen-Nürnberg, Berichte aus der Strömungstechnik, ISBN: 3-8265-9958-6.

[7] Breuer, M. (2005) New Reference Data for the Hill Flow Test Case, personal communication, http://www.hy.bv.tum.de/DFG-CNRS/

[8] Chikhaoui, O., Deng, G., Visonneau, M. (2005) DES Simulations of the Flow Over a Periodic Hill with the ISIS Code, EUROMECH Colloquium 467, Turbulent Flow and Noise Generation, Marseille, France, July 18-20, 2005.

[9] Chikhaoui, O., Deng, G., Visonneau, M. (2005) DES and LES Simulations of the Flow Over a Periodic Hill with the ISIS Code, EUROMECH Colloquium 469, EUROMECH Colloquium 469, Large-Eddy Simulation of Complex Flows, TU Dresden, Germany, Oct. 6-8, 2005.

[10] Deng, G.B., Queutey, P., Visonneau, M. (2005) Three-Dimensional Flow Computation with Reynolds Stress and Algebraic Stress Models, In: Engineering Turbulence Modelling and Experiments 6, ed. W. Rodi and M. Mulas, Elsevier, pp. $389-398$.

[11] Deng, G.B., Visonneau, M. (2003) On the Prediction of Swirling Induced Recirculation, In: Proc. 3th Int. Sym. on Turbulence and Shear Flow Phenomena, Sendai, Japan.

[12] Deng, G.B., Visonneau, M. (1999) Comparison of Explicit Algebraic Stress Models and Second-Order Turbulence Closures for Steady Flows around Ships, In: Proc. 7th Int. Conf. on Numerical Ship Hydrodynamics, Nantes, France.

[13] Ducros, F., Nicoud, F., Poinsot, T. (1998) Wall-Adapting Local Eddy-Viscosity Models for Simulations in Complex Geometries, 6th ICFD Conf. on Numerical Methods for Fluid Dynamics, pp. 293-299.

[14] Durbin, P.A. (1991) Near-Wall Turbulence Closure Modeling Without Damping Functions, Theoretical and Computational Fluid Dynamics, vol. 3, p. 1-13.

[15] Fröhlich, J., Mellen, C.P., Rodi, W., Temmerman, L., Leschziner, M.A. (2005) Highly Resolved Large-Eddy Simulation of Separated Flow in a Channel with Streamwise Periodic Constrictions, J. Fluid Mech., pp. 19-66.

[16] Jakirlić, S., Jester-Zürker, R., Tropea, C. (eds.) (2001) 9th ERCOFTAC/IAHR/COST Workshop on Refined Flow Modeling, Darmstadt University of Technology, Germany, Oct. 4-5, 2001.

[17] Manceau, R., Bonnet, J.-P. (eds.) (2002) 10th Joint ERCOFTAC (SIG-15)/IAHR/QNET-CFD Workshop on Refined Flow Modeling, Université de Poitiers, France, Oct. 10-11, 2002.

[18] Moser, R.D., Kim, J., Mansour N.N. (1999) DNS of Turbulent Channel Flow up to $R e_{\tau}=590$, Physics of Fluids, vol. 11, pp. 943-945. 
[19] Nicoud, F., Ducros, F. (1999) Subgrid-Scale Stress Modelling Based on the Square of the Velocity Gradient Tensor, Flow, Turbulence and Combustion, vol. 62, pp. 183-200.

[20] Piomelli, U., Chasnov, J.R. (1996) Large-Eddy Simulations: Theory and Applications. In: Turbulence and Transition Modelling, (M. Hallbäck, D.S. Henningson, A.V. Johansson and P.H. Alfredson (eds.), pp. 269-331, Kluwer.

[21] Pope, S.B. (2000) Turbulent Flows. Cambridge University Press.

[22] Rhie, C.M., Chow, W.L. (1983) Numerical Study of the Turbulent Flow past an Airfoil with Trailing-Edge Separation, AIAA Journal, vol. 21, pp. 1525-1532.

[23] Rodi, W., Mansour, N.N., Michelassi, V. (1993) One-Equation Near-Wall Turbulence Modeling with the Aid of Direct Simulation Data, J. Fluids Engineering, vol. 115, pp. 196-205.

[24] Sagaut, P. (2001) Large Eddy Simulation for Incompressible Flows - An Introduction. Springer.

[25] Schumann, U. (1975) Subgrid-Scale Model for Finite-Difference Simulations of Turbulent Flows in Plane Channels and Annuli, J. Conputational Physics, vol. 18, pp. 376-404.

[26] Spalart, P.R., Allmaras, S.R. (1994) A One-Equation Turbulence Model for Aerodynamic Flows, La Recherche Aérospatiale, vol. 1, pp. $5-21$.

[27] Spalart, P.R. (2000) Strategies for Turbulence Modeling and Simulations, Int. J. Heat and Fluid Flow, vol. 21, pp. $252-263$.

[28] Temmerman, L., Leschziner, M.A., Mellen, C.P., Fröhlich, J. (2003) Investigation of Wall-Function Approximations and Subgrid-Scale Models in Large Eddy Simulation of Separated Flow in a Channel with Streamwise Periodic Constrictions, Int. J. Heat and Fluid Flow, vol. 24, pp. 157-180.

[29] Yoshizawa, A., Horiuti, K. (1985) A Statistically-Derived Subgrid-Scale Kinetic Energy Model for the Large-Eddy Simulation of Turbulent Flows, J. Phys. Soc. Japan., vol. 54(8), pp. 2834-2839. 